\title{
Full-length transcriptome profiling reveals insight into the cold response of two kiwifruit genotypes (A. arguta) with contrasting freezing tolerances
}

Shihang Sun ${ }^{1,2 \dagger}$, Miaomiao Lin ${ }^{1 \dagger}$, Xiujuan $\mathrm{Qi}^{1}$, Jinyong Chen ${ }^{1}$, Hong Gu${ }^{1}$, Yunpeng Zhong ${ }^{1}$, Leiming Sun ${ }^{1}$, Abid Muhammad ${ }^{1}$, Danfeng Bai ${ }^{1}$, Chungen $\mathrm{Hu}^{2^{*}}$ and Jinbao Fang ${ }^{1 *}$

\begin{abstract}
Background: Kiwifruit (Actinidia Lindl.) is considered an important fruit species worldwide. Due to its temperate origin, this species is highly vulnerable to freezing injury while under low-temperature stress. To obtain further knowledge of the mechanism underlying freezing tolerance, we carried out a hybrid transcriptome analysis of two A. arguta (Actinidi arguta) genotypes, $\mathrm{KL}$ and $\mathrm{RB}$, whose freezing tolerance is high and low, respectively. Both genotypes were subjected to $-25^{\circ} \mathrm{C}$ for $0 \mathrm{~h}, 1 \mathrm{~h}$, and $4 \mathrm{~h}$.

Results: SMRT (single-molecule real-time) RNA-seq data were assembled using the de novo method, producing 24,306 unigenes with an N50 value of 1834 bp. Kyoto Encyclopedia of Genes and Genomes (KEGG) enrichment analysis of DEGs showed that they were involved in the 'starch and sucrose metabolism', the 'mitogen-activated protein kinase (MAPK) signaling pathway', the 'phosphatidylinositol signaling system', the 'inositol phosphate metabolism', and the 'plant hormone signal transduction'. In particular, for 'starch and sucrose metabolism', we identified 3 key genes involved in cellulose degradation, trehalose synthesis, and starch degradation processes. Moreover, the activities of beta-GC (beta-glucosidase), TPS (trehalose-6-phosphate synthase), and BAM (betaamylase), encoded by the abovementioned 3 key genes, were enhanced by cold stress. Three transcription factors (TFs) belonging to the AP2/ERF, bHLH (basic helix-loop-helix), and MYB families were involved in the lowtemperature response. Furthermore, weighted gene coexpression network analysis (WGCNA) indicated that beta-GC, TPS5, and BAM3.1 were the key genes involved in the cold response and were highly coexpressed together with the CBF3, MYC2, and MYB44 genes.
\end{abstract}

\footnotetext{
* Correspondence: chungen@mail.hzau.edu.cn; fangjinbao@caas.cn

† Shihang Sun and Miaomiao Lin contributed equally to this work.

${ }^{2}$ Key Laboratory of Horticultural Plant Biology (Ministry of Education), College

of Horticulture and Forestry Science, Huazhong Agricultural University,

Wuhan 430070, China

${ }^{1}$ Key Laboratory for Fruit Tree Growth, Development and Quality Control,

Zhengzhou Fruit Research Institute, Chinese Academy of Agricultural

Sciences, Zhengzhou 450009, China
}

(C) The Author(s). 2021 Open Access This article is licensed under a Creative Commons Attribution 4.0 International License, which permits use, sharing, adaptation, distribution and reproduction in any medium or format, as long as you give appropriate credit to the original author(s) and the source, provide a link to the Creative Commons licence, and indicate if changes were made. The images or other third party material in this article are included in the article's Creative Commons licence, unless indicated otherwise in a credit line to the material. If material is not included in the article's Creative Commons licence and your intended use is not permitted by statutory regulation or exceeds the permitted use, you will need to obtain permission directly from the copyright holder. To view a copy of this licence, visit http://creativecommons.org/licenses/by/4.0/. The Creative Commons Public Domain Dedication waiver (http://creativecommons.org/publicdomain/zero/1.0/) applies to the data made available in this article, unless otherwise stated in a credit line to the data. 
Conclusions: Cold stress led various changes in kiwifruit, the 'phosphatidylinositol signaling system', 'inositol phosphate metabolism', 'MAPK signaling pathway', 'plant hormone signal transduction', and 'starch and sucrose metabolism' processes were significantly affected by low temperature. Moreover, starch and sucrose metabolism may be the key pathway for tolerant kiwifruit to resist low temperature damages. These results increase our understanding of the complex mechanisms involved in the freezing tolerance of kiwifruit under cold stress and reveal a series of candidate genes for use in breeding new cultivars with enhanced freezing tolerance.

Keywords: Low temperature, Kiwifruit, Freezing tolerance, Full-length transcriptome, Cold stress

\section{Background}

Low temperature is one of the main abiotic stresses affecting horticultural plant development and growth and also restricts the geographical distribution of plants [1, 2]. Because plants are sessile organisms, low temperature can lead to decreased cell membrane fluidity and water potential and loss of protein function, resulting in enormous amounts of damage [3]. However, throughout their long-term evolutionary development, plants have evolved a set of complex mechanisms to adapt to low temperatures [4]. Freezing tolerance (FT) is considered an essential ability of plants to survive low-temperature stress and is an adaptive process during which the expression of proteins related to transcriptional regulation and functional materials is induced $[5,6]$. In addition, complex metabolic regulatory activity is involved in FT, and metabolites such as antioxidant compounds (SOD, ascorbic acid), osmolytes (raffinose, trehalose) and nitrogenous compounds (glutathione and glycine betaine) have positive effects on increasing FT [7-9].

Genome data are basic information that can be used to facilitate research on FT mechanisms [10]. The A. chinensis (Actinidia chinensis) genome data were published in 2013; the genome has an $\sim 140 \times$ sequencing depth, and the size of the current assembly is $616.1 \mathrm{Mb}$, with a total of 39,040 genes [11]. There are recently published kiwifruit genome assemblies for A. chinensis 'Red 5' and Actinidia eriantha 'White' as well as an improved assembly of 'Hongyang', all of which are diploid [12-14]. However, A. arguta is a tetraploid species, and its genome information is unknown due to genome complexity resulting from the polyploidy conditions. When genome data are absent, SMRT (single-molecule real-time) RNAseq data can act as a useful tool to substitute for genomic [15]. SMRT long-read sequencing technology is currently becoming a popular method to obtain information on full-length (FL) mRNA molecules and has been extensively used for transcriptome profiling in various plant species [16-19]. FL transcript sequences that avoid incorrect assembly can show explicit information on each gene [20]. However, the SMRT method cannot calculate the mapped number of reads to quantify the expression level of genes, but this problem can be solved with NGS (next-generation sequencing) technology [21].
Furthermore, the SMRT sequencing method has a high error rate (up to $15 \%$ ) for a single base, which can be corrected with NGS reads. Hence, hybrid RNA sequencing technology is a useful tool to obtain both qualitative and quantitative transcriptome results [22].

Transcriptome analysis has become an effective technology to characterize molecular regulatory activity and investigate the genes involved in response to various abiotic stresses, such as drought stress, waterlogging stress, and salt stress [23-25]. However, studies on transcriptional level changes under cold stress in kiwifruit are limited. In model plant species (Arabidopsis), previous studies have identified and confirmed several of the key genes and transcription factors (TFs) involved in the response to low-temperature stress [26]. Among these TFs, bHLH, MYB, and AP2/ERF TFs are considered important in terms of plant cold-responsive processes [2729]. Many studies have shown the important roles of transcription factors, including CBFs (C-repeat binding factors), ICEs (inducers of CBF expression genes), and MYB15, in cold regulation and acclimation [30]. CBFs recognize and bind to cis-elements in the promoters of COR (cold-responsive) genes, thus triggering gene expression. At present, researchers have characterized several COR genes, including ZAT, COR15a, and KIN [31]. Moreover, metabolic pathways such as those of sugar metabolism, flavonoid metabolism, and amino acid metabolism are important in the response to cold stress [32-34]. Due to limited knowledge of the complex FT mechanism in kiwifruit, we planned to identify key pathways in kiwifruit through the transcriptome method.

A. arguta, unlike other Actinidia species, has an extensive geographic distribution varying from $25^{\circ} \mathrm{N}$ to $50^{\circ} \mathrm{N}$. Moreover, these kiwifruit species have different FTs against cold stress. Especially in northern China, $A$. arguta has a remarkable FT, which implies that some changes in genetic mechanisms have occurred to enhance the FT of $A$. arguta [35]. However, studies on the FT mechanism in $A$. arguta are scarce. In this study, using SMRT sequencing and NGS analysis, we investigated two kiwifruit genotypes, $\mathrm{KL}$ and $\mathrm{RB}$, with high and low FT, respectively. The physiological parameters of both genotypes were analyzed to strongly support the transcriptome analysis results. The present study aimed 
to identify differentially expressed genes and pathways involved in the FT of kiwifruit. Our results will reveal useful genes to develop new freezing-tolerant cultivars, which is important for coping with growing problems under low temperature for the kiwifruit industry in the near future.

\section{Results}

\section{Physiochemical characteristics of kiwifruit under cold} stress

The shoots of two kiwifruit genotypes with contrasting freezing tolerance (KL: freezing-tolerant; RB: freezingsensitive) were subjected to $-25^{\circ} \mathrm{C}$ for $0 \mathrm{~h}, 1 \mathrm{~h}$ and $4 \mathrm{~h}$. The anatomical structural changes of the shoots were observed under the microscope. The anatomical structure of the shoots was not significantly different under cold stress in either genotype (Fig. 1a). Due to the relationship between anatomical structure and freezing tolerance (FT), we further investigated several indices of both untreated genotypes. There were several differences in both genotypes, including in the ratio of pith, vessel density, ratio of xylem, and ratio of phloem. The ratio of pith and the ratio of xylem in KL were higher than those in $\mathrm{RB}$, whereas the vessel density and ratio of phloem in RB were higher than those in KL (Fig. 1b, c). These differences suggested that geographical distribution led to anatomical structural changes in both genotypes.

Two physiological indices, MDA content and $\mathrm{H}_{2} \mathrm{O}_{2}$ content, were measured to evaluate the FT of kiwifruit under cold stress. The plasma membrane has an important role in plants; however, low temperatures always lead to lipid peroxidation of the membrane. Membrane lipid peroxidation generates a set of metabolites, one of which is MDA. The MDA content in KL and RB showed a significant change from $0 \mathrm{~h}$ to $4 \mathrm{~h}$ (Fig. 2a). The MDA content in $\mathrm{KL}$ increased by approximately $13 \%$ from $0 \mathrm{~h}$ $(15.8 \mu \mathrm{mol} / \mathrm{g})$ to $4 \mathrm{~h}(17.9 \mu \mathrm{mol} / \mathrm{g})$ at $-25^{\circ} \mathrm{C}$, whereas that in $\mathrm{RB}$ increased by approximately $26 \%$ from $0 \mathrm{~h}$ $(17.9 \mu \mathrm{mol} / \mathrm{g})$ to $4 \mathrm{~h}(22.5 \mu \mathrm{mol} / \mathrm{g})$ at $-25^{\circ} \mathrm{C}$. Moreover, cold stress accelerated the accumulation of $\mathrm{H}_{2} \mathrm{O}_{2}$ in plants. The content of $\mathrm{H}_{2} \mathrm{O}_{2}$ in $\mathrm{KL}$ increased by 2-fold from $0 \mathrm{~h}(3.4 \mu \mathrm{mol} / \mathrm{g})$ to $1 \mathrm{~h}(6.9 \mu \mathrm{mol} / \mathrm{g})$ and then

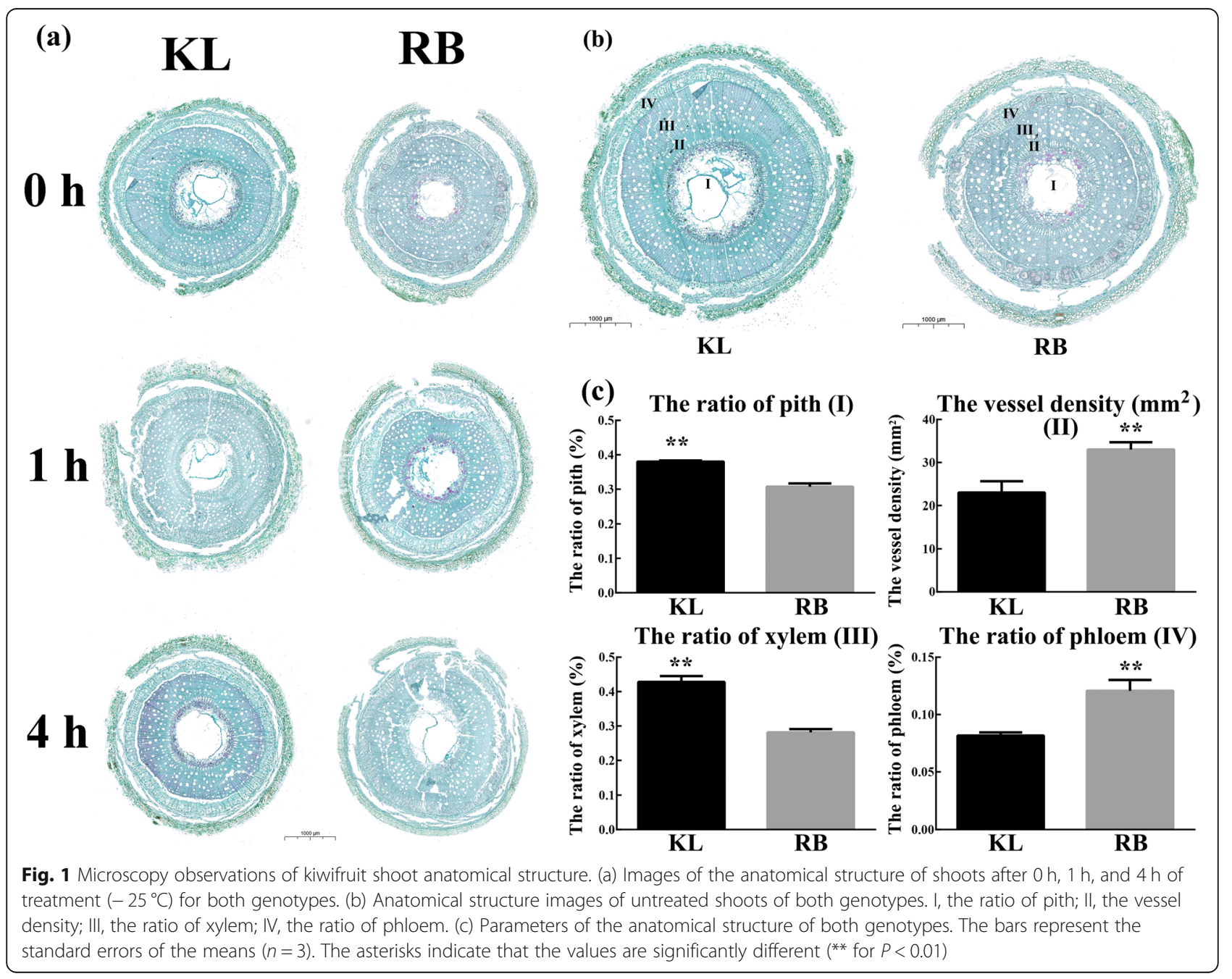




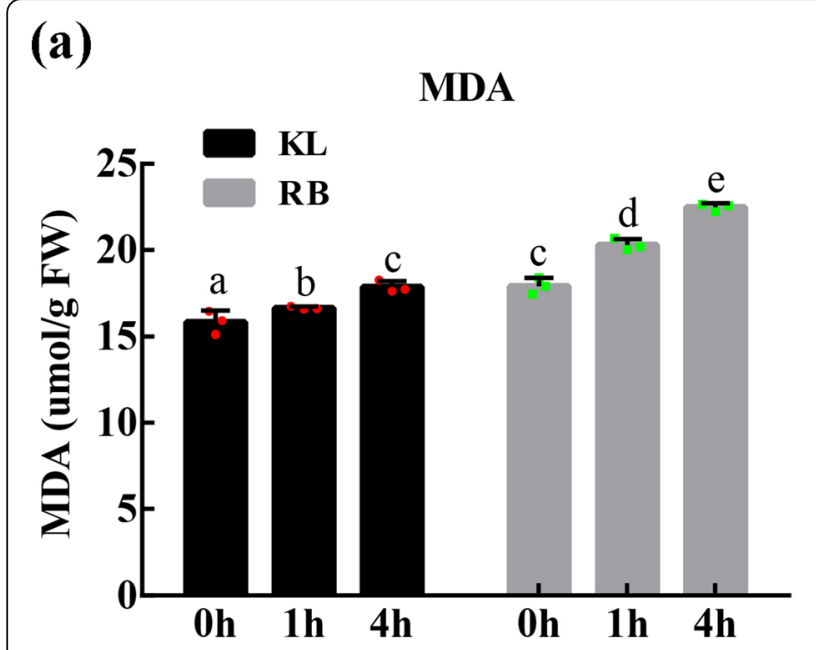

(b)

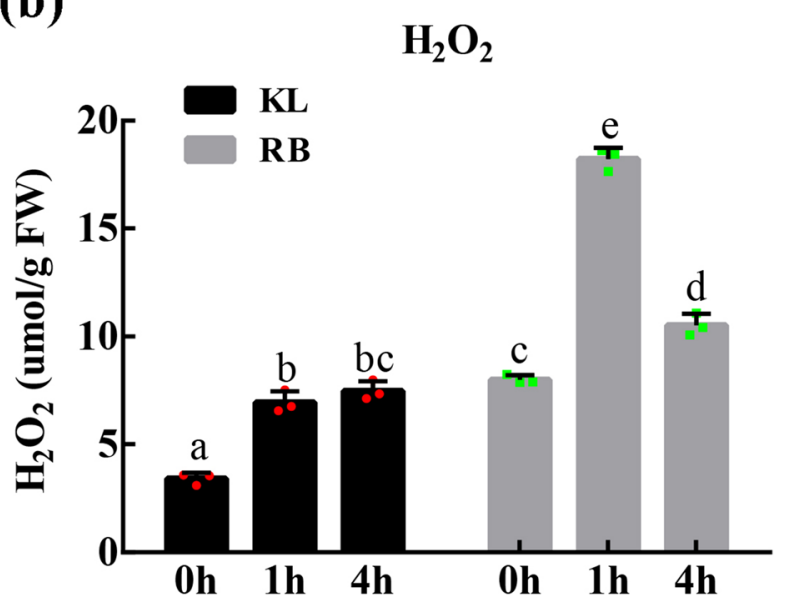

Fig. 2 Comparative physiological characterization of kiwifruit shoots of two different genotypes. (a) Malondialdehyde (MDA) content in the shoots. (b) Hydrogen peroxide $\left(\mathrm{H}_{2} \mathrm{O}_{2}\right)$ content in the shoots. The experiments included three biological replicates. Multiple comparisons were performed for significant differences $(P<0.05$, two-way ANOVA and Tukey's test; the error bars represent the SDs)

showed no significant change at $4 \mathrm{~h}(7.4 \mu \mathrm{mol} / \mathrm{g})$; the content was consistent with that at $1 \mathrm{~h}$. However, the $\mathrm{H}_{2} \mathrm{O}_{2}$ content in $\mathrm{RB}$ increased by 2.3-fold from $0 \mathrm{~h}$ $(7.9 \mu \mathrm{mol} / \mathrm{g})$ to $1 \mathrm{~h}(18.2 \mu \mathrm{mol} / \mathrm{g})$ and then declined at 4 h $(10.5 \mu \mathrm{mol} / \mathrm{g})$ (Fig. $2 \mathrm{~b})$. Taken together, these results indicated that the membrane stability and level of oxidative stress in kiwifruit were affected by cold stress. Moreover, the damage that KL experienced from lowtemperature stress was lower than that of RB.

\section{Summary of NGS and SMRT transcriptome sequencing of kiwifruit}

NGS transcriptome sequencing generated 906,672,934 raw reads and 866,664,476 clean reads (95.59\%, 130.01 $\mathrm{Gb}$ ) with an average Q30 value of $93.70 \%$ and an average GC content of $45.54 \%$ (Table 1). SMRT transcriptome sequencing generated 22,604,106 subreads $(32.37 \mathrm{~Gb}$ in size, with an average length of $1388 \mathrm{bp}$ and an N50 value of $1834 \mathrm{bp}$ ), that were corrected using the NGS data (Fig. 3a). A total of 350,711 circular consensus sequences (CCSs) were obtained from the filtration of the subreads. The number of full-length nonchimeric (FLNC) reads was 254,139 , whose average length was $1602 \mathrm{bp}$.

\section{Functional annotation of unigenes}

To determine the possible functions of unigenes in kiwifruit, a total of 24,306 unigenes in the SMRT transcriptome were functionally annotated using five databases (the NR, GO, KEGG, KOG, and SwissProt databases). All the unigenes had an approximately $98 \%$ annotation rate in at least one database [NR $(23,796,97.90 \%)$, GO $(15,833,65.14 \%)$, KEGG $(12,201,50.20 \%)$, KOG $(15,584$, 64.12\%), and SwissProt (20,715, 85.23\%)] (Fig. 3b).
To determine the conservation level of the unigene sequences of kiwifruit in other plant species, the unigene sequences in kiwifruit were queried in the NCBI NR database (Fig. 3c). The unigene sequences in kiwifruit displayed the highest similarity to sequences from $A$. chinensis (86.57\%), followed by those from Camellia sinensis (2.90\%), Quercus suber (2.02\%), Actinidia deliciosa (0.75\%), Vitis vinifera (0.30\%), Actinidia kolomikta

Table 1 Summary of the transcriptome data from PacBio RSII platform and Illumina Hiseq platform

\begin{tabular}{ll}
\hline Platform & \\
\hline PacBio RSII & 350711 \\
Total CCS & 271699 \\
Full length reads & 254139 \\
FLNC reads & $1602 \mathrm{bp}$ \\
Average FLNC read length & $32.37 \mathrm{~Gb}$ \\
Subreads base (G) & 22604106 \\
Subreads number & 1388 \\
Average subreads length & 1834 \\
N50 & 24306 \\
Total unigenes & 1953 \\
TF number & \\
Illumina Hiseq & 50370718.56 \\
Raw reads (average) & 48148026.44 \\
Clean reads (average) & $130.01 \mathrm{~Gb}$ \\
Clean base (G) & $0.03 \%$ \\
Base error rate (average) & $93.70 \%$ \\
Q30 (average) & $45.54 \%$ \\
GC content (average) &
\end{tabular}



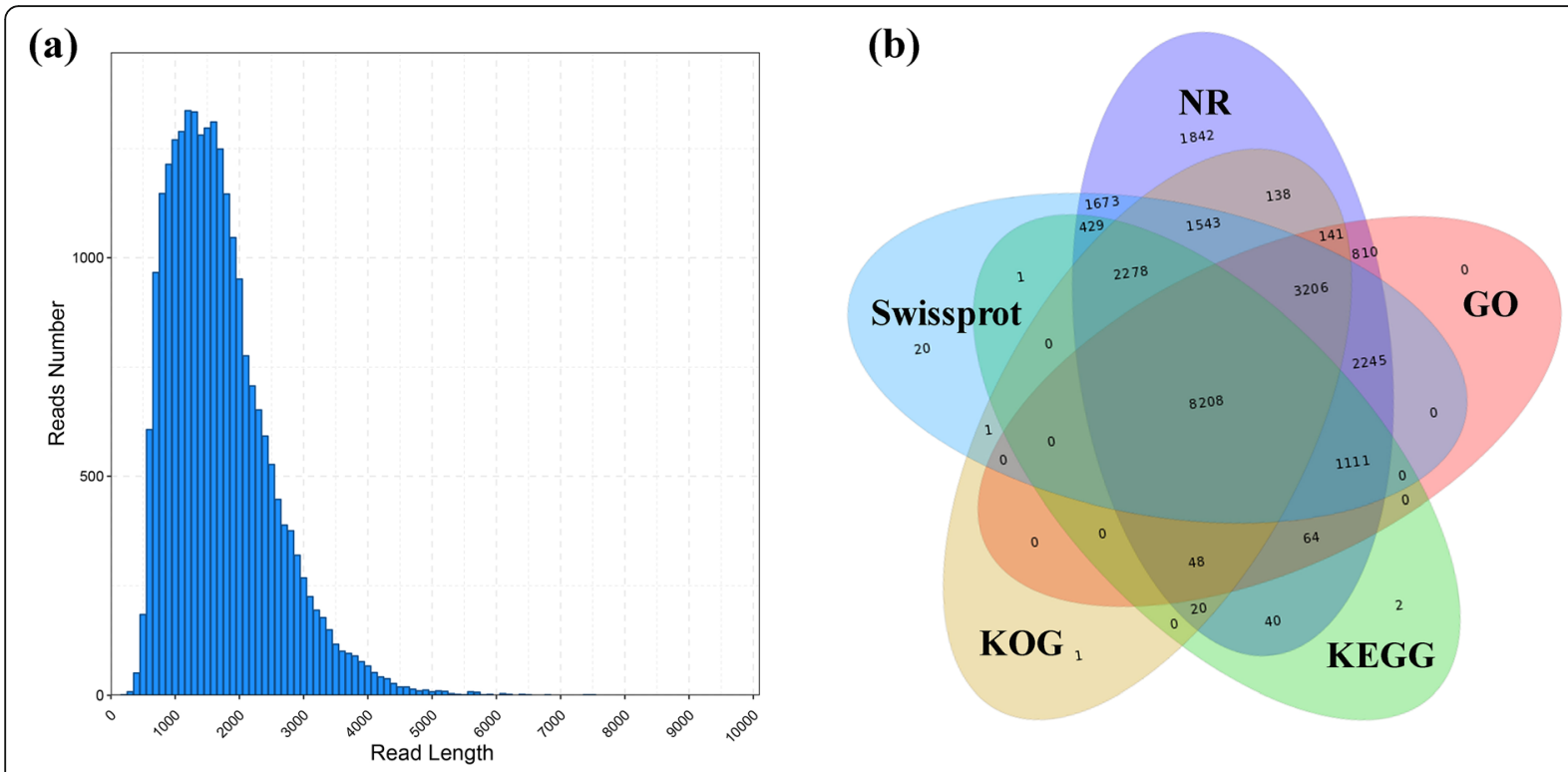

(c)

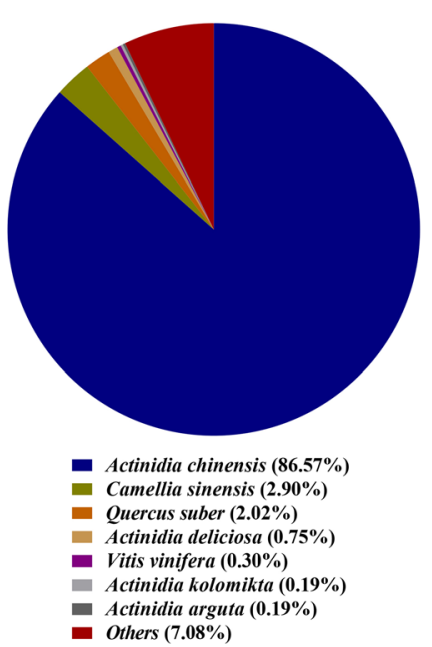

(d)

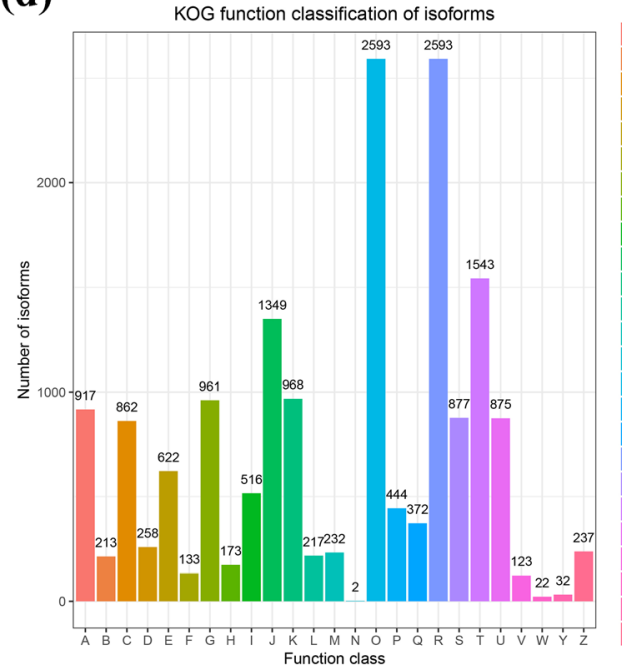

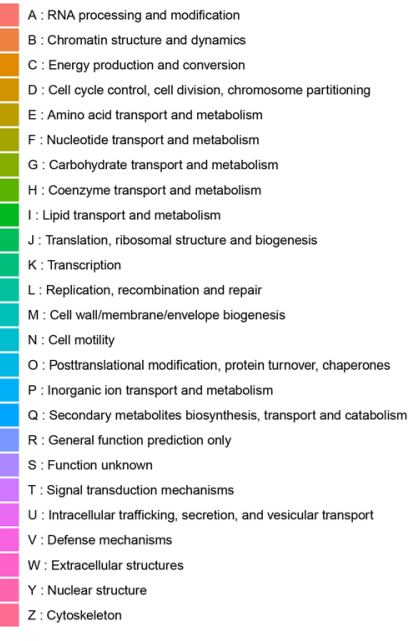

Fig. 3 Overview of SMRT sequencing results and annotation of unigenes. (a) Distribution of full-length reads. (b) Overlap between the number of all unigenes according to five databases. (c) Distribution of unigene annotations based on the NR database for the species distribution statistics. (d) KOG functional classification of all unigenes

(0.19\%), and $A$. arguta (0.19\%). The results suggested that the majority of unigenes were annotated in $A$. chinensis.

All the kiwifruit unigenes were subsequently queried against the KOG database (Fig. 3d). A total of 15,584 sequences were annotated, with 26 functional categories. 'Posttranslational modification, protein turnover, and chaperones' (2593, 16.64\%); 'general function prediction only' (2593, 16.64\%); and 'signal transduction mechanisms' $(1543,9.90 \%)$ were the top three categories. Furthermore, 'cell motility' (2, 0.012\%) was the smallest category with the fewest unigenes.
A total of 15,833 unigenes were annotated in the GO database (Fig. S1), which were successfully clustered into 48 functional groups, of which 14 categories belonged to BPs (biological processes), 22 belonged to CCs (cellular components), and 12 belonged to MFs (molecular functions). We also conducted an analysis based on KEGG pathways to obtain key information, including that on intracellular metabolic pathways and biological functions of genes in kiwifruit (Fig. S2). A total of 12,201 unigene sequences were clustered into 19 KEGG pathway categories. Moreover, the most significant category among these pathways was 'carbohydrate metabolism' (1446; 
$11.85 \%)$, followed by 'translation' (1406; 11.52\%) and then 'folding, sorting and degradation' (1310; 10.73\%).

\section{Identification of DEGs}

DEGs in the two contrasting genotypes were screened from a combination of NGS transcriptome data and SMRT transcriptome data. The expression levels of DEGs were compared between every two groups based on the FPKM values (Fig. 4). There were 473 DEGs (367 up- and 106 downregulated) between KL$0 \mathrm{~h}$ and KL-1 h, 579 DEGs (510 up- and 69 downregulated) between KL- $0 \mathrm{~h}$ and KL-4h, 866 DEGs (742 up- and 124 downregulated) between RB- $0 \mathrm{~h}$ and RB$1 \mathrm{~h}, 925$ DEGs (750 up- and 175 downregulated) between RB-0 $h$ and RB-4h, 10,300 DEGs (5028 upand 5272 downregulated) between RB- $0 \mathrm{~h}$ and KL-0 h, 10,539 DEGs (4980 up- and 5559 downregulated) between RB-1 $\mathrm{h}$ and KL-1 h, and 10,603 DEGs (5127 up- and 5476 downregulated) between RB-4h and $\mathrm{KL}-4 \mathrm{~h}$. These results indicated that the number of DEGs increased after low-temperature treatment in both genotypes. Moreover, RB exhibited more DEGs than did $\mathrm{KL}$ at $1 \mathrm{~h}$ and $4 \mathrm{~h}$ of treatment. For example, the number of DEGs in RB after $1 \mathrm{~h}$ of treatment was 866, which was almost twice that in KL DEGs (473). These differences in DEG numbers indicated that cold stress could induce more dynamic transcriptomic changes in $\mathrm{RB}$ than in $\mathrm{KL}$ and that $\mathrm{KL}$ had a stronger ability to maintain stable transcriptomes under cold stress. Furthermore, among the 3 time points, $4 \mathrm{~h}$ was the specific treatment time point that had the most abundant DEGs.

\section{KEGG classification of DEGs}

The DEGs identified in KL between $0 \mathrm{~h}$ and $4 \mathrm{~h}$ were queried in the KEGG pathway database (Fig. 5a). KEGG pathway enrichment showed that the most significant subcategory among a series of pathways was 'starch and sucrose metabolism', followed by the 'MAPK signaling pathway', the 'phosphatidylinositol signaling system', 'inositol phosphate metabolism', and 'arginine and proline metabolism' in KL. However, KEGG analysis of RB between $0 \mathrm{~h}$ and $4 \mathrm{~h}$ showed that 'starch and sucrose metabolism' was involved in the response to cold stress (Fig. 5b). For the 'starch and sucrose metabolism' pathway, 11 transcripts were involved in this pathway, while most (9 out of 11) transcripts continuously had higher expression in KL than in RB. For the 'MAPK signaling pathway', 13 out of 18 transcripts involved in the MAPK signaling pathway had higher expression in KL than in RB. For the 'phosphatidylinositol signaling system' pathway, 5 of 8 transcripts had higher expression in KL than in RB. For the 'inositol phosphate metabolism' pathway, 2 transcripts of 4 transcripts had higher expression in $\mathrm{KL}$, and for the 'arginine and proline metabolism' pathway, 4 of 5 transcripts had higher expression in KL (Table S1) (Fig. 5c). Taken together, these results highlighted the involvement of different pathways in the response to low-temperature stress.

\section{Identification of DEGs related to phytohormones and signaling}

Among the DEGs, we identified several that were associated with phytohormones and calcium signaling. Seventeen DEGs involved in phytohormone pathways were selected, and their expression patterns are illustrated in

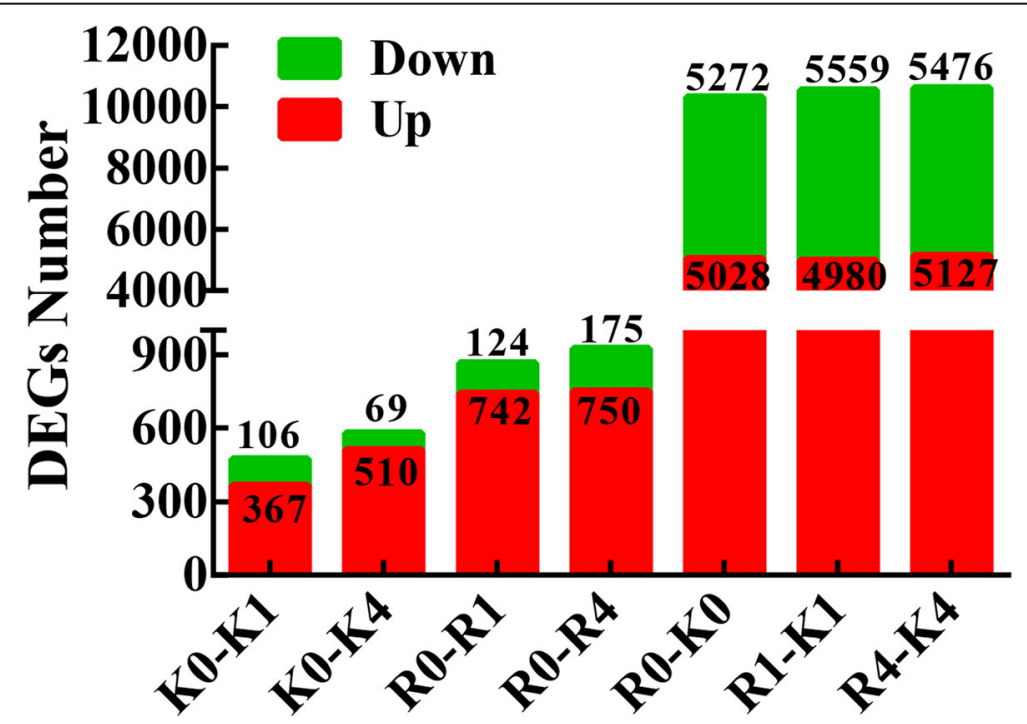

Fig. 4 Summary of differential expression analysis of kiwifruit under cold stress. The numbers of upregulated and downregulated DEGs were identified in each comparison of different time intervals. KO, KL-0 h; K1, KL-1 h; K4, KL-4 h; RO, RB-0 h; R1, RB-1 h; R4, RB-4 h 


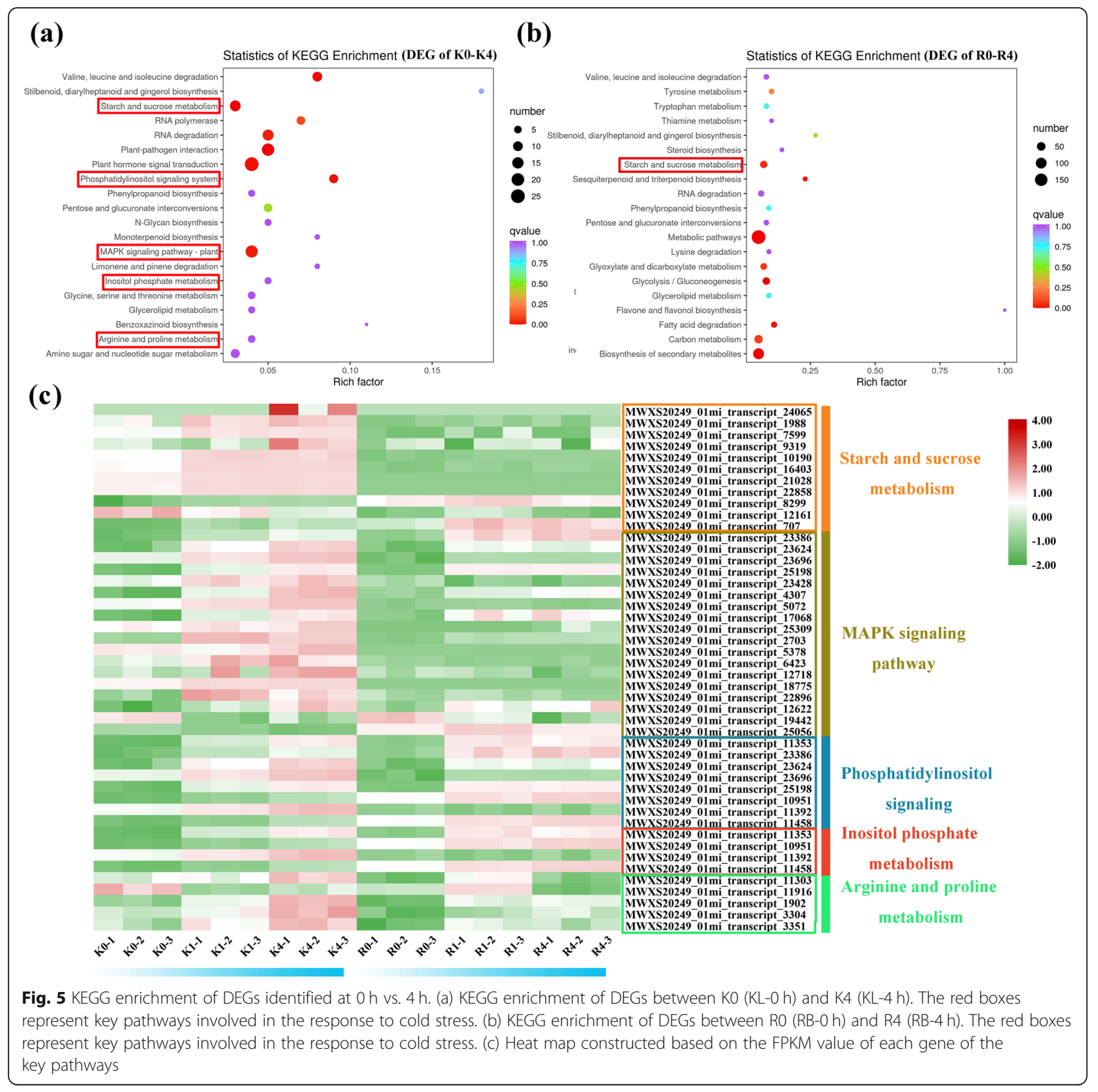

Fig. 6a; these DEGs included 10 genes involved in the ethylene signaling process, 5 genes involved in the jasmonic acid signaling process, and 2 genes involved in the brassinosteroid signaling process (Table S2). For the ethylene process, 9 out of 10 genes were induced by cold stress in $\mathrm{KL}$; these genes were expressed at levels higher than those in RB. The abovementioned 9 genes encoded ERF, EIN, and EBF proteins. For the jasmonic acid signaling process, 3 out of 5 genes had higher expression in $\mathrm{KL}$ than in RB; the above 3 genes encoded MYC2. For the brassinosteroid process, both genes had different expression levels in the two genotypes, and there were no changes in $\mathrm{KL}$, whereas cold stress decreased the expression of 2 genes encoding TCH4 (xyloglucan endotransglucosylase) in RB. Moreover, a total of 96 transcripts encoded calcium signaling-related genes, including 1 CRLK (calcium/calmodulin-regulated receptor-like kinase), 14 CAMTAs (calmodulin-binding transcription activators), 22 CIPKs (CBL-interacting protein kinases), 46 CDPKs (calcium-dependent protein kinases), and 12 CNGCs (cyclic nucleotide/calmodulin-gated ion channels). Seventeen genes were significantly induced by cold stress in KL; however, 5 genes were significantly induced by low temperature in RB; the 12 genes with specific 
(a)

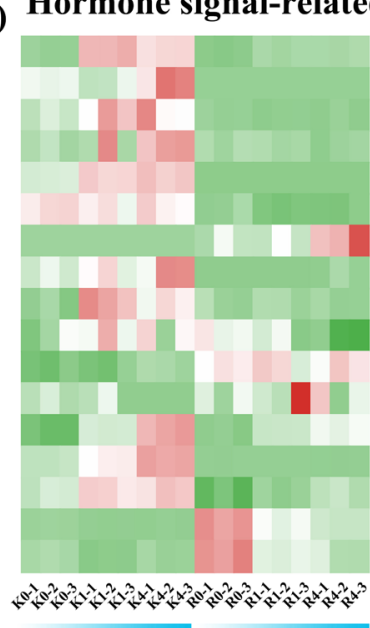

(b)

MWXS20249_01mi_transcript_2703 MWXS20249_01mi_transcript_5378 MWXS20249_01mi_transcript_6423 MWXS20249_01mi_transcript_12718 MWXS20249_01mi_transcript_18775 MWXS20249_01mi_transcript_10740 ETH MWXS20249 01mi transcript 2878 MWXS20249_01mi_transcript_25309 MWXS20249_01mi_transcript_22896 MWXS20249_01mi_transcript_24087 MWXS20249_01mi_transcript_19976 MWXS20249_01mi_transcript_20227 MWXS20249_01mi_transcript_4307 JA MWXS20249_01mi_transcript_5072 MWXS20249_01mi_transcript_3652 MWXS20249_01mi_transcript_21568 MWXS20249_01mi_transcript_25860

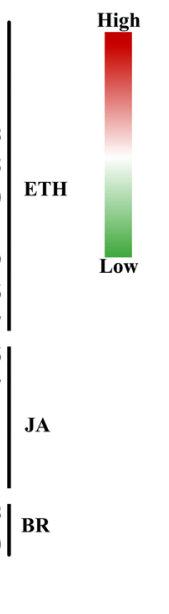

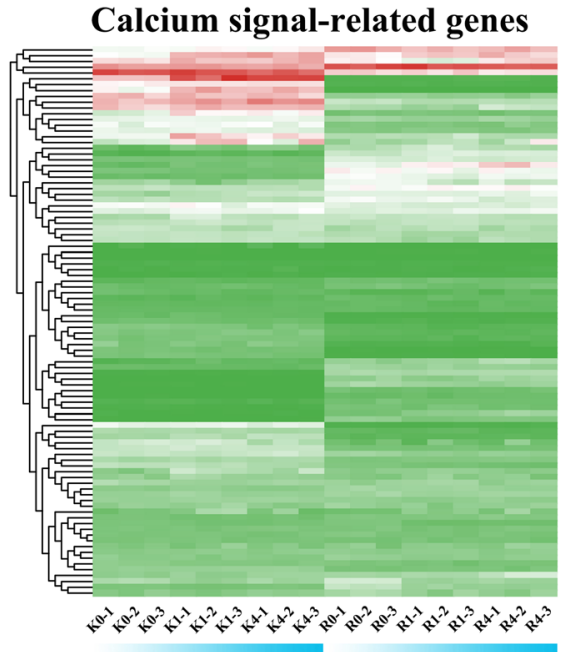

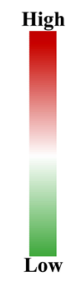

Fig. 6 Heat map of signal transduction-related genes in cold-stressed kiwifruit. (a) Heat map constructed based on hormone signal-related genes. (b) Heat map constructed based on calcium signal-related genes. Ethylene, ETH; jasmonic acid, JA; brassinolide, BR expression in KL included 1 CAMTA, 5 CIPK genes, 2 CDPK genes, and 4 CNGC genes (Fig. 6b).

\section{Identification of DEGs related to starch and sucrose metabolism and measurement of sugar metabolites}

A comparison between the KL- $4 \mathrm{~h}$ and $\mathrm{RB}-4 \mathrm{~h}$ groups was performed. A KEGG enrichment scatterplot of the DEGs was constructed to provide a graphical presentation of the KEGG enrichment analysis results (Fig. 7a). The 'starch and sucrose metabolism' pathway, which was strongly enriched, was selected and presented. There were 3 significant metabolic processes in 'starch and sucrose metabolism': 'cellulose degradation', 'trehalose synthesis', and 'starch degradation'. Nine DEGs were involved in the above 3 significant metabolic processes. Among these 9 functional genes, those encoding SS (sucrose synthase), CBH1 (cellobiohydrolase), betaglucosidase (beta-GC), TPS, hexokinase, ADP-glucose pyrophosphorylase, SS (starch synthase), SBE (starch branching enzyme), and beta-amylase (BAM) were highly expressed in KL compared with RB (Fig. 7b). Therefore, we speculated that starch degradation, trehalose synthesis, and cellulose degradation played an important role in enhancing FT.

The beta-GC, TPS, and BAM enzymes are key enzymes involved in 'cellulose degradation', 'trehalose synthesis', and 'starch degradation' processes, respectively. These enzymes were affected by cold stress but showed different changes in activity in both genotypes under cold stress (Fig. 8a, c, e). Under cold stress, beta-GC activity in KL showed an initial decrease and then an increase. However, in RB, beta-GC activity showed no significant change. Cold stress caused an increase in TPS activity. In KL, TPS activity remained relatively high; in contrast, cold stress suppressed TPS activity in the cold period in RB. BAM activity increased under cold stress in $\mathrm{KL}$, whereas it decreased in RB. In addition, the BAM activity in $\mathrm{KL}$ was higher than that in $\mathrm{RB}$ throughout the measured period.

The level of soluble sugar in kiwifruit shoots was also measured. Cold stress increased the soluble sugar content throughout the cold treatment in KL, and there was no significant difference in the soluble sugar content between the $4 \mathrm{~h}$ and $0 \mathrm{~h}$ treatments in RB (Fig. 8b). As shown in Fig. 8d, KL showed increased trehalose content at $4 \mathrm{~h}$, whereas RB showed no significant difference throughout the cold treatment. Under cold stress, cold increased the maltose content in KL but decreased it in RB (Fig. 8f). These results indicated that kiwifruit actively stimulated a set of starch metabolic pathways to enhance FT to resist freezing damage during the low-temperature treatment periods. In addition, low temperature may promote soluble sugar, trehalose and maltose accumulation under cold stress to increase FT.

\section{TFs in response to low-temperature stress}

Transcription factors play a key role in cold stress and are involved in transcriptional regulatory networks. A total of 20 categories of TF families were identified (Fig. 9a). The DEGs encoding most of the TFs were involved in the low-temperature response, and the majority belonged to the ERF, bHLH, and MYB families. Members of 3 kinds of TF families have been previously identified to regulate FT. Consequently, DEGs derived from three transcription factor families (ERF, bHLH, and MYB) are shown in Fig. 9b, c, d. The 16 TFs (Table S3) were screened, 5 of which belonged to the ERF, 5 


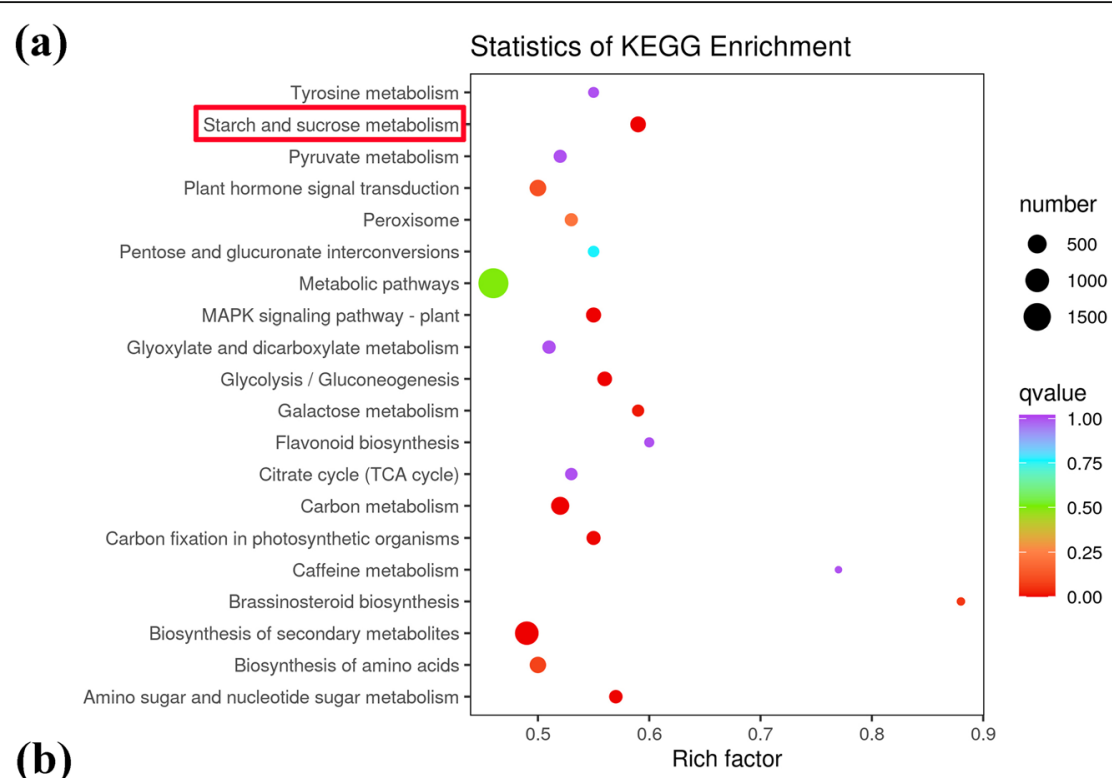

(b)

\section{Sucrose}

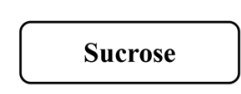

synthase
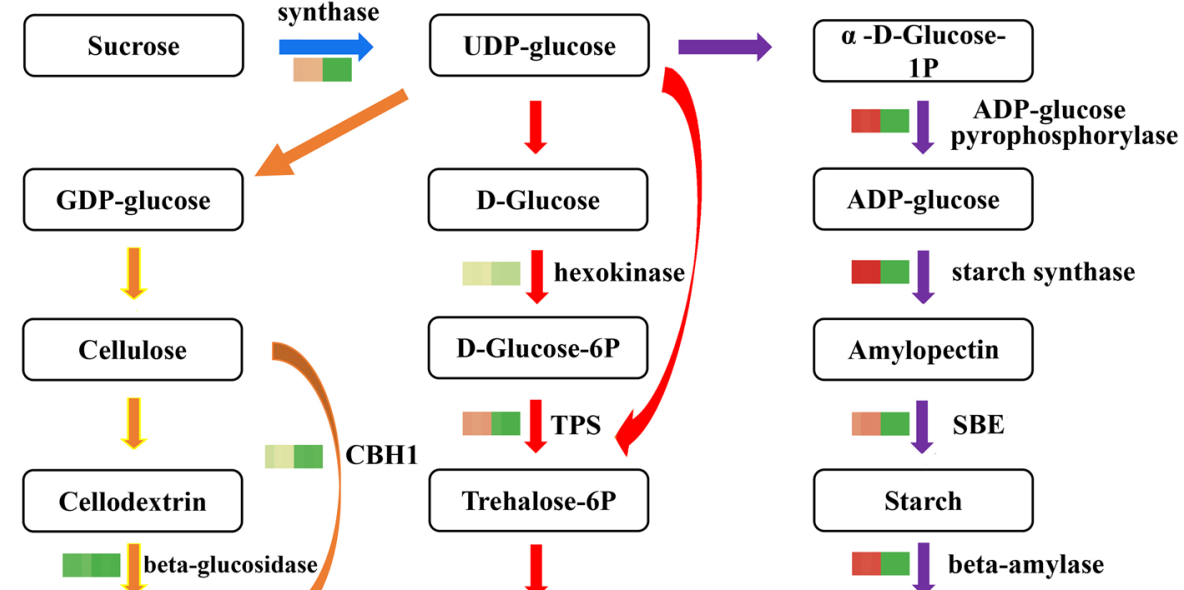

Cellobiose
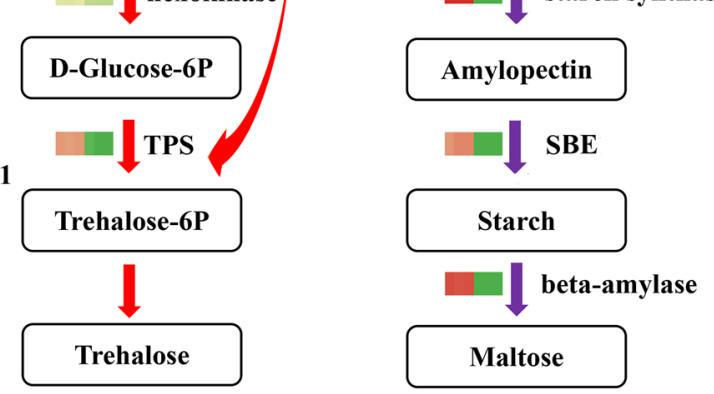

Relative expression Left:K4 Right:R4

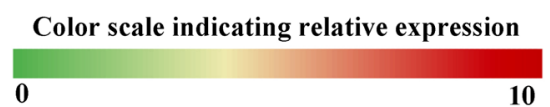

Fig. 7 (a) KEGG enrichment of DEGs between $\mathrm{KL}-4 \mathrm{~h}$ and RB-4 h. The red box represents the key pathway involved in the response to cold stress. (b) DEGs involved in the starch and sucrose metabolism pathways. Gene expression is represented by the grids under the $4 \mathrm{~h}$ treatment, with FPKM values of both genotypes shown

belonged to the bHLH, and 6 belonged to the MYB families. The 5 AaERF genes and 5 AaMYC genes had higher expression in KL than in $\mathrm{RB}$, and 6 AaMYB genes had lower expression in KL than in RB. In addition to the abovementioned ERF, bHLH, and MYB family members, 4 genes belonging to the $\mathrm{C} 2 \mathrm{H} 2$ family encode zinc finger proteins (ZAT6, ZAT10, and ZAT12), which had higher expression in $\mathrm{KL}$ than in RB; other members of TFs, including 1 HSF gene, 1 NAC gene, and 1 WRKY gene, were also significantly increased upon cold stress (Table 2).

\section{Construction of WGCNA networks and identification of candidate genes in kiwifruit}

To determine the correlation between samples and modules, we constructed a gene coexpression network using RNA-seq data. A network heatmap (interconnectivity plot) of the gene network is shown, which displays the resulting modules and corresponding hierarchical clustering dendrograms (Fig. 10a). Seven modules containing 60-251 DEGs each were identified. MDA, $\mathrm{H}_{2} \mathrm{O}_{2}$, betaGC, soluble sugar, TPS, trehalose, BAM, and maltose contents were used as phenotypic data for calculating 


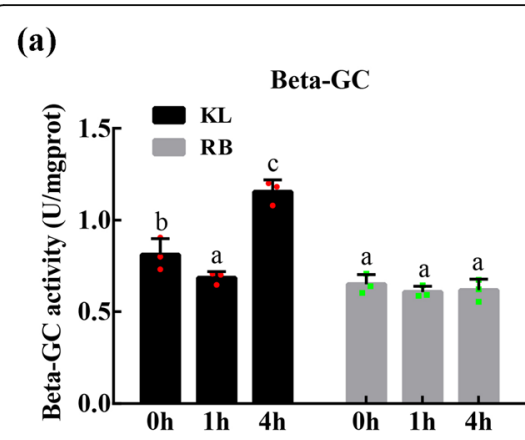

(b)

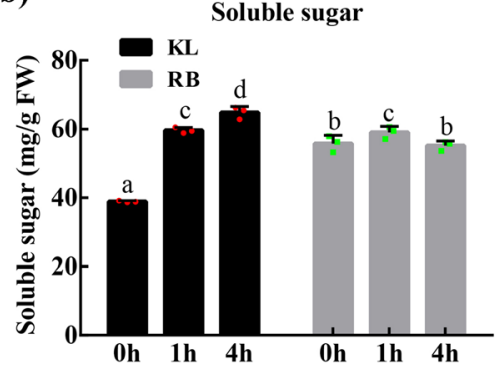

(c)

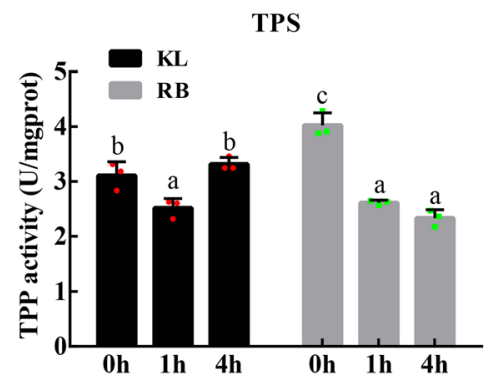

(d)

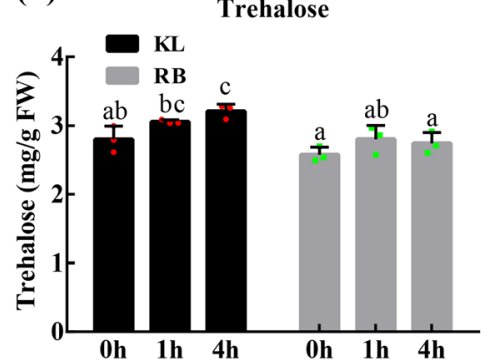

(e)

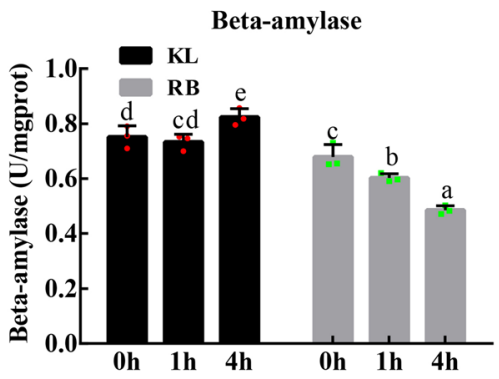

(f)

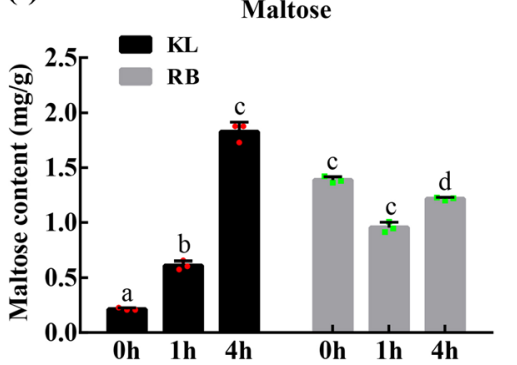

Fig. 8 Changes in beta-GC (a), soluble sugar (b), TPS (c), trehalose (d), beta-amylase (e), and maltose (f) contents in the shoots of both genotypes under cold stress

the relationships in the trait modules. The results of the correlation between the modules and starch-sucrose pathway indicated that the correlation values ranged from -0.85 to 0.92 (Fig. 10b). The eigengenes of the blue modules showed significant correlations with the starch-sugar pathway. The heat map of the blue module showed that FPKM values were higher in KL than in RB (Fig. 10c). To further identify key candidate genes in the blue module, the hub genes in the blue module were calculated using Cytoscape software (Table S4). The results of the hub gene search showed that the connections of the sugar-related and transcription factor-regulated DEGs were in the blue module, and sugar-related genes were highly coexpressed with transcription factorregulated genes (Fig. 10d).

\section{Validation of candidate genes by qRT-PCR}

For further validation of the RNA-seq results and candidate genes linked to starch and sucrose metabolism, we selected 9 DEGs involved in 'starch and sucrose metabolism' and 3 DEGs identified as potential candidate TFs for qRT-PCR experiments. While the fold-change of gene expression levels measured by qRT-PCR and RNAseq were different, the expression patterns of the majority of the 12 DEGs assessed by the two methods showed similar trends, which validated the reliability of the RNA-seq results (Fig. 11). Moreover, these results confirmed that beta-GC, TPS5, BAM3.1, CBF3, MYC2, and $M Y B 44$ were potential candidate genes involved in the regulation of FT (Fig. S3).

\section{Discussion}

Kiwifruit is not only an important fruit species but also an economically important plant species worldwide. Cold stress is an abiotic stress that harms the development of kiwifruit and limits its yield. To enhance FT, it is essential to explore the FT mechanism of kiwifruit. However, the ploidy of kiwifruit is comparatively complex and leads to complex genomic information, and the breeding of cultivars with high FT is obstructed by the absence of functional genomic data. We therefore used a hybrid sequencing method combining both SMRT and NGS sequencing to determine the regulatory network of gene expression and induction in shoots of kiwifruit under cold stress. Moreover, physiological and transcriptome analyses were carried out to determine the FT responses in kiwifruit in this study.

\section{Involvement of starch and sucrose metabolism in high freezing tolerance}

The enzymes and genes participating in starch degradation play an important role in the response to cold stress [36, 37]. In our study, a set of key genes associated with starch and sucrose metabolism were screened, and their expression levels were determined under cold stress. A majority of the identified genes were induced under cold stress, showing that cellulose degradation, trehalose accumulation and starch degradation were activated during this period, which may be important for the improvement of FT of kiwifruit (Fig. 8). Betaglucosidase is a rate-limiting enzyme involved in the 


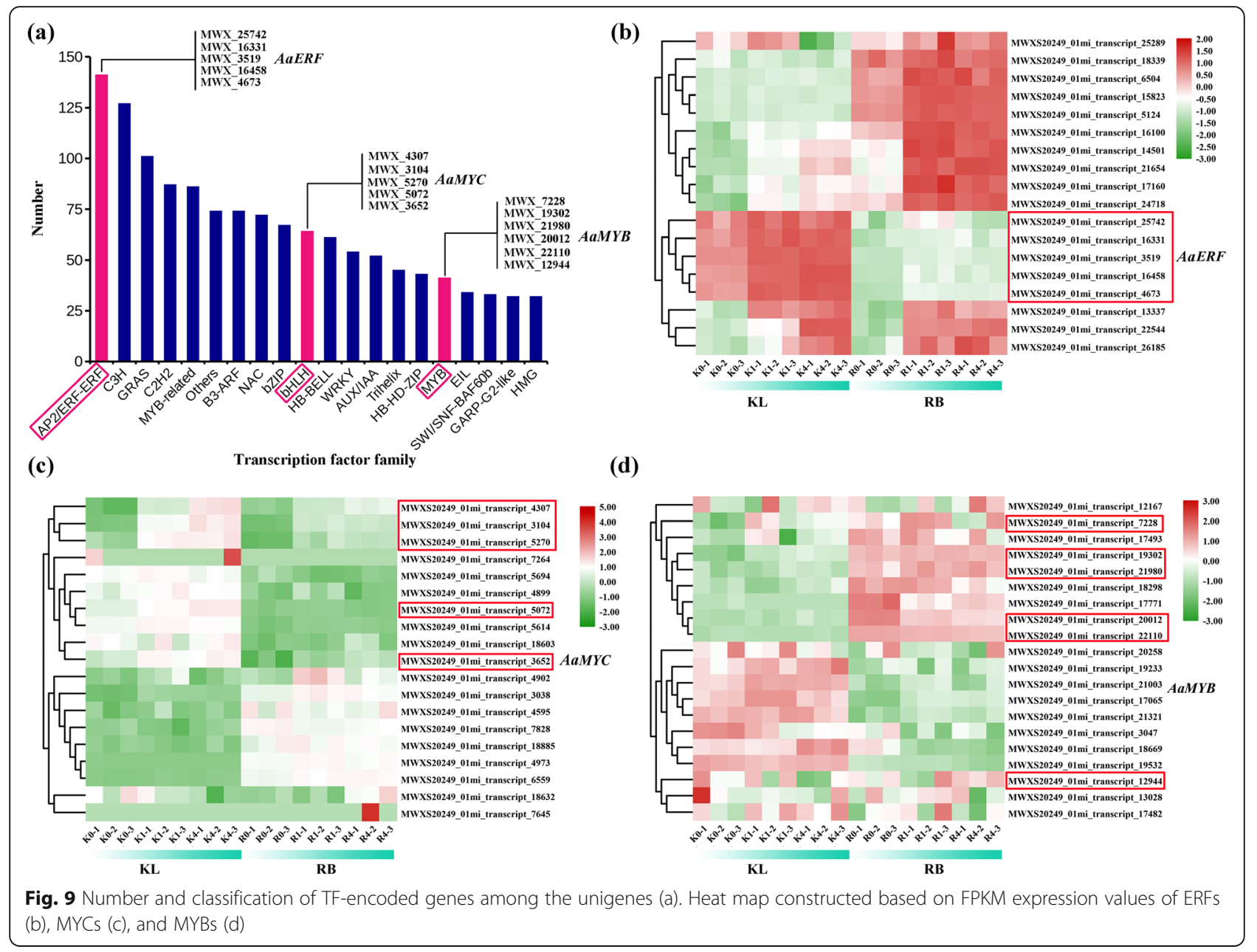

hydrolysis of cellulose [38]. Some beta-glucosidases can affect the structure of the cell wall, which might play a key role in cell adaptations to the physical deformations caused by cold stress [39]. After cold acclimation, A. thaliana plants with a mutation in sfr2-1, which encodes beta-glucosidase, are more sensitive to FT than the wild type is [40]. Under various abiotic stresses, beta-glucosidases hydrolyze inert precursors to release antioxidant substances [41]. A study on the transcriptional analyses of chickpea showed that the beta- glucosidase gene was screened to act as a differentially expressed gene under cold stress and that its expression was induced in response to low temperature [42]. Betaglucosidase BGLU10 may be involved in cold, salt, and drought stress in A. thaliana [43]. In our study, the expression of the beta-glucosidase gene was upregulated under cold stress, and the expression of the betaglucosidase gene in $\mathrm{KL}$ was higher than that in RB. Moreover, beta-glucosidase activity was activated, and

Table 2 FPKM values of differentially expressed TF in both genotypes

\begin{tabular}{|c|c|c|c|c|c|c|c|}
\hline ID & Name & Ko & K1 & K4 & Ro & R1 & R4 \\
\hline MWXS20249_01mi_transcript_23086 & ZAT6 & 27.10 & 127.74 & 169.98 & 6.75 & 15.77 & 17.10 \\
\hline MWXS20249_01mi_transcript_23199 & ZAT10 & 11.69 & 97.55 & 162.67 & 3.30 & 68.27 & 62.87 \\
\hline MWXS20249_01mi_transcript_24340 & ZAT10 & 61.80 & 259.50 & 362.35 & 44.81 & 181.13 & 163.13 \\
\hline MWXS20249_01mi_transcript_25307 & ZAT12 & 0.81 & 14.02 & 30.38 & 0.00 & 0.00 & 0.00 \\
\hline MWXS20249_01mi_transcript_2738 & HSF & 4.87 & 11.46 & 19.73 & 3.87 & 13.35 & 11.02 \\
\hline MWXS20249_01mi_transcript_8860 & NAC & 2.97 & 14.45 & 15.23 & 1.27 & 3.78 & 3.33 \\
\hline MWXS20249_01mi_transcript_4511 & WRKY & 9.24 & 19.70 & 29.89 & 6.20 & 12.51 & 14.13 \\
\hline
\end{tabular}




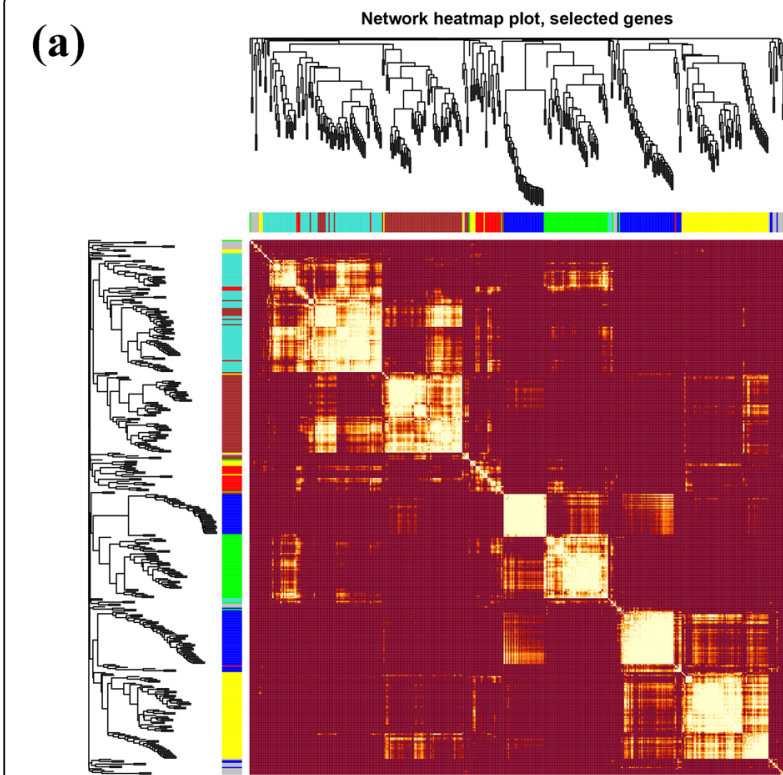

(b)

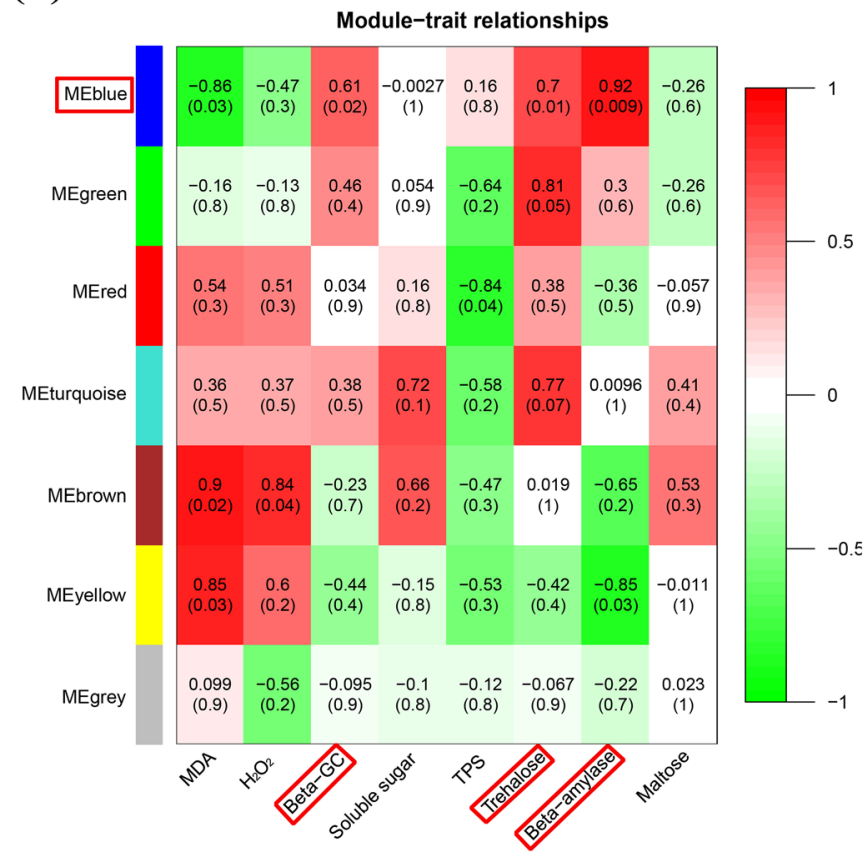

(c)

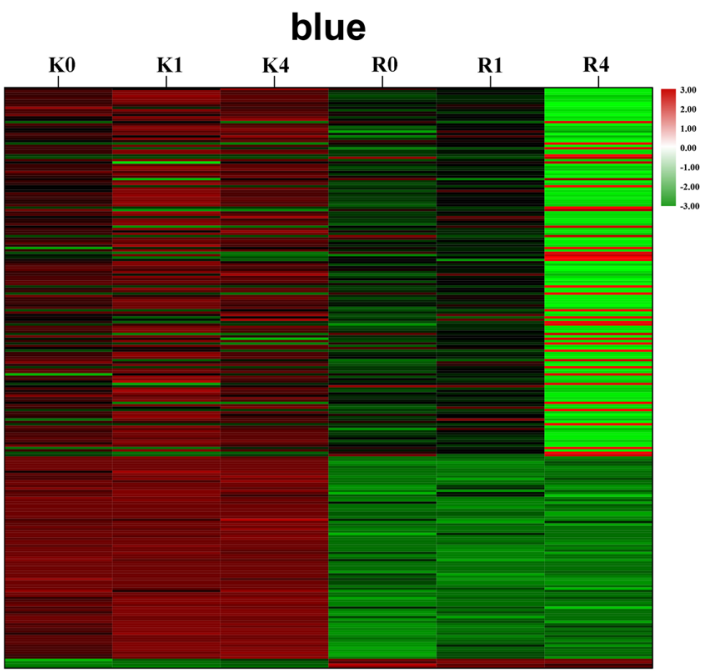

(d)

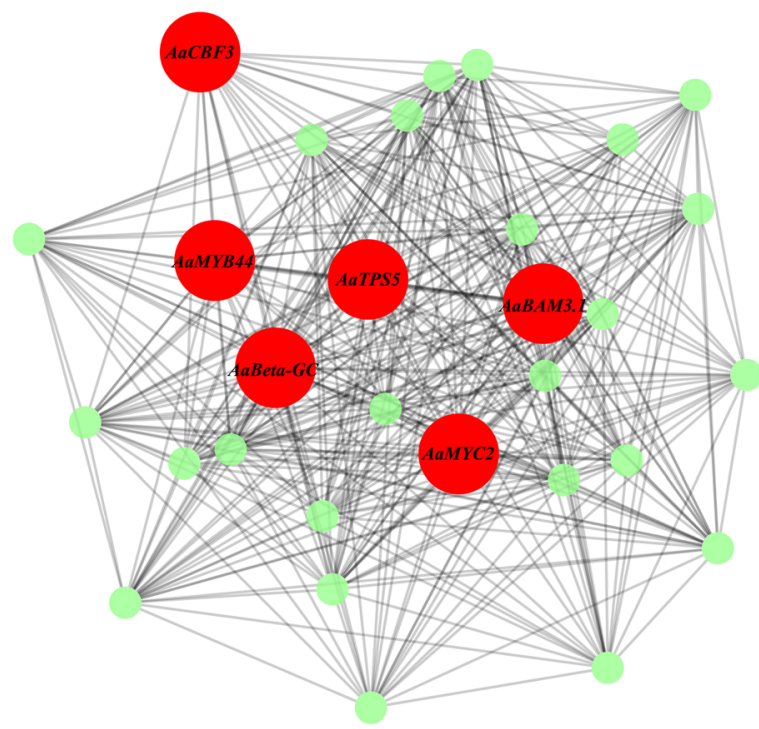

Fig. 10 Identification of coexpression network modules in kiwifruit. (a) Cluster dendrogram and heat map of genes subjected to coexpression module calculations. (b) Module-trait associations based on Pearson correlations. The color key from green to red represents $r^{2}$ values from - 1 to 1, respectively. (c) Heat map constructed for the blue module genes. (d) Gene network of the blue module, which is positively correlated with beta-GC $\left(r^{2}=0.61\right)$, trehalose $\left(r^{2}=0.7\right)$, and BAM $\left(r^{2}=0.92\right)$ contents. The candidate genes within the blue module are highlighted in red due to the higher weight within the module and coded for gene descriptors based on annotations

the soluble sugar content also increased under cold stress.

Additionally, trehalose, a multifunctional disaccharide, exists extensively in various plant species $[44,45]$. Trehalose forms a special protective structure on the surface of the cell membrane, protects the activity of functional protein molecules, and thus resists multiple abiotic stresses [46]. Both trehalose-6-phosphate and trehalosephosphate-phosphatase play an important role in the synthesis of trehalose. Rice plants overexpressing OsTPS1 and OsTPP1 can acquire higher FT [47, 48]. TPS is the crucial controller, and there are 11 copies of the TPS gene in $A$. thaliana. Transgenic plants of $A$. thaliana overexpressing TPP and TPS increased the 


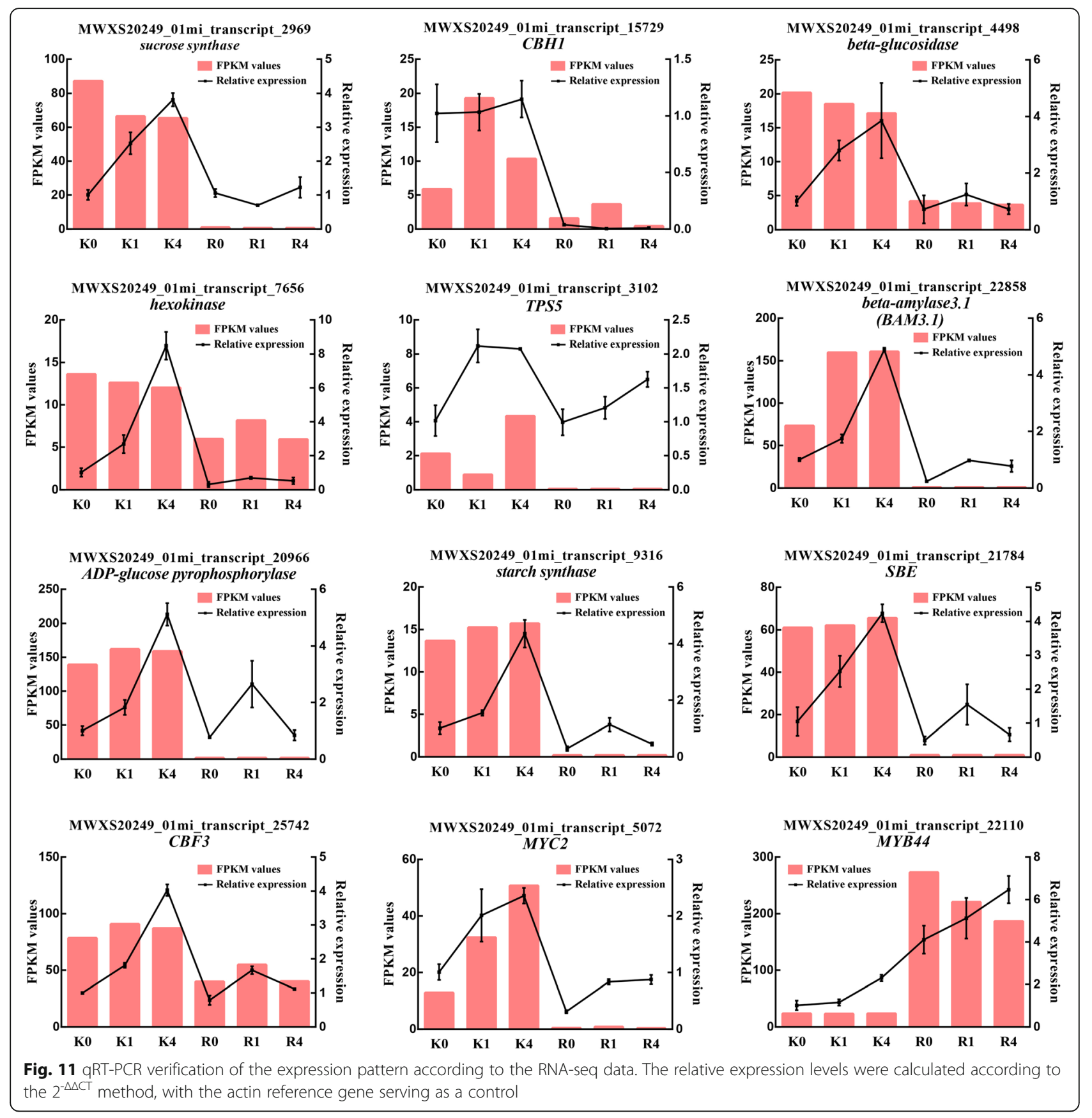

tolerance ability under low-temperature and salt stress [49]. Exogenous trehalose can induce the expression of stress-related genes in plants [50]. In this study, TPS genes were isolated and upregulated under cold stress. Moreover, OsICE1 can serve as a positively regulated TF to activate the expression of OSTPP [51]; likewise, we identified that both $M Y C 2$ and TPS were induced under cold stress in KL.

BAM is an important hydrolase that degrades starch to yield maltose and has a key effect on FT improvement [52]. The expression and activity of BAM were reported to be significantly induced after a $6 \mathrm{~h}$ treatment of cold stress in A. thaliana [53]. In kiwifruit, AaBAM3 was upregulated to increase the activity of beta-amylase from autumn to winter in China [54]. In pear, researchers have found the same results as those in kiwifruit [55]. Interestingly, in Poncirus trifoliata, there is a CBFbinding element in the promoter of PtrBAM1, and CBF can regulate the expression of PtrBAM1, which plays a role in FT by regulating the sugar metabolite content [56]. In our study, the results of kiwifruit were similar to those of other plant species, and $B A M$ was upregulated 
under cold stress. In general, the abovementioned 3 genes may be key genes that enhance the FT of KL.

\section{Pathways involved in cold signaling}

The phosphatidylinositol signaling system and the inositol phosphate metabolic pathways have been reported to be associated with FT $[57,58]$. The significant KEGG pathways during the $4 \mathrm{~h}$ treatment were enriched in both of these pathways in KL but not in RB (Fig. 5a, b). These results of the KEGG analyses showed that the gene expression levels of the different genotypes were different under low-temperature stress. Inositol 3-phosphate (IP3), which is generated by phospholipase C-mediated hydrolysis of phosphatidylinositol bisphosphate (PIP2), triggers a set of cellular processes by releasing calcium. These releases can increase calcium from low to high levels. Calcium also acts as a secondary messenger to transduce cold signals. In $A$. thaliana, the phosphatidylinositol-hydrolyzing phospholipase gene was upregulated under multiple abiotic stresses $[59,60]$. Moreover, myo-inositol-3-phosphate synthase activity was shown to be activated during the ABA signaling response in Spirodela polyrhiza [61]. Overexpression of Dmyo-inositol-3-phosphate synthase leads to elevated levels of inositol in Arabidopsis under salt stress [62]. In our study, three transcripts (MWXS20249_01mi_transcript_10951, MWXS20249_01mi_transcript_11392, MWXS20249_01mi_transcript_11458) encoding myotubularin phosphatases were identified; these proteins subject phosphatidylinositol to different phosphorylation patterns, resulting in the production of distinct phosphatidylinositol phosphates. The genes encoding myotubularin phosphatases were induced under cold stress to increase FT. Inositol-1,3,4-trisphosphate 5/6-kinase (MWXS20249_01mi_transcript_11353) is involved in inositol phosphate metabolism, which can also trigger signal transduction. Hence, KL might enhance its FT by activating genes related to both the phosphatidylinositol signaling system and the inositol phosphate metabolic pathways.

Plant hormones play an important role in regulating stress responses and activating downstream lowtemperature pathways. ETH, JA, and BR hormones are vital stress hormones involved in the modulation of the responses to cold stress. We noticed that several ETH signaling-related genes, such as ERF, EIN, and EBF, were upregulated during cold stress in the freezing-tolerant genotype (KL). Both the stress-responsive JA signalingrelated genes $J A Z$ and $M Y C$ were induced under cold stress in KL (Fig. 6a); relatedly, PtrMYC2 of Poncirus trifoliata integrates JA signals to modulate cold-induced GB accumulation by directly regulating the expression of PtrBADH-l, a betaine aldehyde dehydrogenase (BADH)like gene [9]. BR signaling is also the key pathway controlling the response to cold stress, and brassinazoleresistant 1 (BZR1) was shown to be upregulated under cold stress in Poncirus trifoliata to enhance its FT [63]. Furthermore, the TCH4 gene acts downstream of BZR1 [64]. In our study, we identified two TCH4 genes, and these genes were repressed under cold stress in RB; however, no change in their expression was found in KL. Both TCH4 genes might be the key reason for the decrease in FT of the sensitive genotype (RB).

The members of several kinase families are involved in cold signal transduction, including CDPK, CIPK, CRLK, CAMTA, and CNGC. The members of these gene families play an important role in regulating FT. In our study, we identified several DEGs, including 2 CDPK genes, 5 CIPK genes, 1 CAMTA gene, and 4 CNGC genes, indicating that the FT of kiwifruit might be enhanced through the $\mathrm{Ca}^{2+}$ signaling pathway. Interestingly, recent research in rice showed that cyclic nucleotide-gated channel (OsCNGC9) positively regulates chilling tolerance by mediating cytoplasmic calcium elevation [65]. In KL, 4 GNGC genes had higher expression than that in RB. These GNGC genes may be candidate genes for explaining the difference in FT between the genotypes.

The MAPK signaling pathway was also significantly induced, showing that this pathway is associated with cold stress responses [66]. Calcium/calmodulin binds to CRLK1 and activates it, after which CRLK1 interacts with MEKK1 (mitogen-activated protein kinase kinase kinase 1), leading to MAPK activation and freezing tolerance. MAPK cascades transduce cold signals into intracellular responses using various classes of protein kinases. With respect to kinases, some genes encoding MPKs (mitogen-activated protein kinases) were shown to be upregulated in response to low temperature in $A$. thaliana [67]. Some researchers identified another MAP3K that played an important role in cold stress signal transduction to enhance FT in Poncirus trifoliata [68]. In our study, a transcript (MWXS20249_01mi transcript_12622) encoding MPK3/6 was identified, and its expression increased under cold stress. Coldactivated MPK3/6 phosphorylates ICE1 proteins to improve their stability and transcriptional activity, which consequently regulates $C B F$ expression in and FT of plants.

\section{Transcriptional regulatory pathways involved in the cold response}

TFs, e.g., AP2/ERF, bHLH, and MYB, are differentially expressed at low temperature and have been reported to be associated with FT in A. thaliana. Moreover, the members of the AP2/ERF TF family were the most abundant TFs according to our data. The $C B F$ gene belongs to the AP2/ERF family; this gene can induce the 
expression of cold-responsive genes (CORs) that are involved in multiple abiotic stress responses [69]. Previous studies have indicated that AtCBF1, 2, and 3 are responsive to cold stress in A. thaliana. Overexpression of the $C B F$ gene was shown to activate the expression of COR genes and increase the FT of plants. In our study, the CBF1 gene was identified as a candidate gene for enhancing the FT of KL, and its upstream regulated genes, including CAMTA, EBF, EIN, ZAT10, and ZAT12, were also induced under cold stress in KL (Table 2). Members of the bHLH family also have a positive function in enhancing FT. bHLH TFs, which act upstream of $C B F$, can bind to the G/E-box motif of the $C B F$ gene and subsequently improve FT by activating COR genes $[70,71]$. Previous studies have shown that overexpression of ICE improves the FT of $A$. thaliana. According to our data, five $M Y C 2$ genes belonging to the bHLH family were upregulated in $\mathrm{KL}$, whereas there were no significant changes in RB (Fig. 9c). MYB transcription factors may play an important role in the low-temperature response and changes in the expression levels of COR genes, affecting the physiological response to resist adverse environmental conditions. For example, AtMYB15 is a transcription regulatory gene that negatively regulates the FT of Arabidopsis [72]. MYBs were differentially expressed between KL and RB under cold stress, implying a key role of $M Y B$ genes in the low-temperature response under cold stress (Fig. 9d). Overall, TFs are crucial mediators in regulating the expression of COR genes to affect the FT of kiwifruit.

\section{WGCNA of the gene network regulation of the FT mechanism}

Freezing tolerance is the result of a complex biochemical pathway that is involved in the regulation of multiple genes; therefore, a single gene is not enough to explain FT. Genes involved in multiple metabolic pathways are usually regulated through coordinated expression, so correlation models are often used to identify gene coexpression networks [73, 74]. KEGG analysis and TF analysis revealed several candidate genes that respond to cold stress in kiwifruit in our study. We obtained 7 gene expression modules via WGCNA, of which one module was strongly correlated with starch-sucrose metabolism. The blue module from the WGCNA correlated significantly with beta-GC, TPS, and BAM. Through hub gene searching in the blue module, beta-GC, TPS5, BAM3.1, $C B F 3, M Y C 2$, and MYB44 were screened as key genes. These results indicated that the above 6 candidate genes were key players in the cold response in kiwifruit. In general, the overview of these genes using coexpression networks would strengthen our understanding of coordinated regulation of multiple genes involved in the FT of kiwifruit.
Based on our results, we hypothesized that when kiwifruit are subjected to cold stress, various metabolites act as low-temperature sensors, after which cells release calcium through phosphatidylinositol signaling and inositol phosphate metabolism. Calcium interacts with CRLK to transduce cold signals through the MAPK signaling pathway, and then the MAPK signaling pathway triggers signal transduction via TFs such as ERF, bHLH, and MYB TFs. Moreover, plant hormones including ETH, JA, and BRs also serve as signaling molecules to regulate COR gene expression. Subsequently, the starch-sucrose pathway, which regulates osmolyte and redox balance, and the production of COR genes, including beta-GC, TPS, and beta-amylase, through activation of the CBF pathway and non-CBF pathway, in turn enhance FT. Overall, the accumulation of soluble sugars, trehalose, and maltose plays an important role in resisting adverse temperature conditions in kiwifruit. A diagram explaining the process involving kiwifruit FT is shown in Fig. 12.

Our results indicated that signaling transduction pathway and starch and sucrose pathway were key pathways to regulate kiwifruit freezing tolerance and that key genes involved in these pathways might lead to different FT between freezing tolerant genotype and sensitive genotype. However, one limitation in our study should also be considered: keeping in mind the limited number of samples (two samples) in current study, comparative transcriptome of two genotypes might lead to identify fake DEGs which were not related to freezing adaption but to different genetic background. Therefore, firstly, the two materials were compared with their respective untreatment materials (control) to sort out low temperature induced transcripts when we screened the differential transcripts. Secondly, transcriptome is a feasible method to identify key genes, the qRT-PCR results of key candidate genes validate authentic DEGs. Moreover, it is better method to find key genes through genome-wide association study (GWAS) or bulksequencing-analysis (BSA) after the genome of $A$. arguta is sequenced. In future studies, larger and more diverse samples should be investigated to further identify key DEGs. Additionally, the relationship of TFs and 3 subpathways including starch degradation, trehalose synthesis and cellulose degradation need to be clearly confirmed. Future studies also need to focus on how certain changes in the hub gene can affect other pathways or traits.

\section{Conclusions}

In the present study, an integrated transcriptome profile of contrasting kiwifruit genotypes under low temperature was analyzed via RNA-seq combined with SMRT and NGS technologies to reveal the FT 


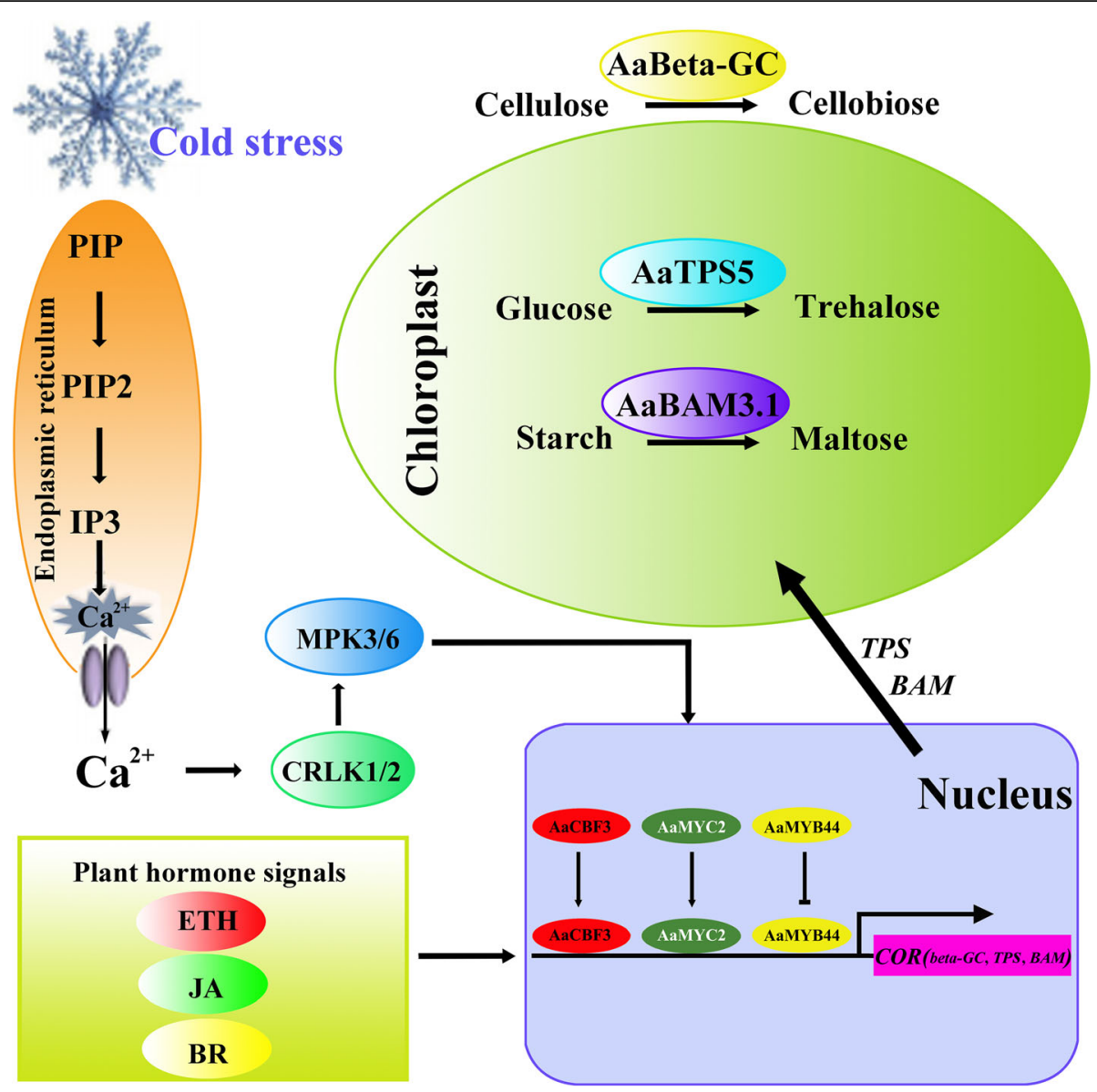

Fig. 12 Putative candidate model of gene involvement in the FT of kiwifruit. Phosphatidylinositol phosphate, PIP; phosphatidylinositol bisphosphate, PIP2; inositol 3-phosphate IP3; ethylene, ETH; jasmonic acid, JA; brassinolide, BR

mechanism. We identified the following genes in response to low temperature: (1) starch and sucrose metabolism-related genes (beta-GC, TPS5, BAM3.1) and (2) transcription factor genes (CBF3, MYC2, MYB44). Additionally, the 'phosphatidylinositol signaling system', 'inositol phosphate metabolism', 'MAPK signaling pathway', 'plant hormone signal transduction', and 'starch and sucrose metabolism' processes were involved in the cold response. These processes might play an important role in the responses to low temperature in kiwifruit. Our results provide novel viewpoints on a set of molecular mechanisms of kiwifruit FT. In future work, we will concentrate on the functional verification of the structural genes beta-GC, which is related to cellulose degradation, TPS, which is related to trehalose synthesis, and $B A M$, which is related to starch degradation, and explore how they work in response to cold stress.

\section{Methods}

\section{Plant materials and cold treatment}

Two A. arguta (Sieb. et Zucc.) Planch. et Miq. genotypes, 'Kuilv male' (KL) and 'Ruby-3' (RB), were collected in the national kiwifruit germplasm repository of Zhengzhou Fruit Research Institute, CAAS, Henan Province, China. KL originated from Jilin Province $\left(125^{\circ} \mathrm{E}, 44^{\circ} \mathrm{N}\right.$; China), and RB originated from Henan Province $\left(113^{\circ} \mathrm{E}\right.$, $34^{\circ} \mathrm{N}$; China). Tissue culture-generated seedlings were planted in 3-L pots and then grown for 3 years. Oneyear-old shoots were collected in early January 2020, and then the detached shoots were put into a freezer in darkness. Three biological replicates of shoots for RNA sequencing (RNA-seq) were collected randomly from all plants of each genotype under $-25^{\circ} \mathrm{C}$ treatment at $0 \mathrm{~h}$, $1 \mathrm{~h}$, and $4 \mathrm{~h}$. At the same time, shoots were collected for measurement of physiological indices. The collected samples were frozen immediately in liquid nitrogen and stored in a $-80^{\circ} \mathrm{C}$ freezer for further study.

\section{Histological, physiological and biochemical analyses}

The anatomical structure of the shoots was observed by the paraffin wax method for both genotypes [75]. In brief, the shoot samples were immediately submerged in FAA (5\% formaldehyde:6\% acetic acid:63\% alcohol:26\% $\mathrm{H}_{2} \mathrm{O}$ ) fixative solution for $72 \mathrm{~h}$. The shoot samples were 
then gradually dehydrated using an alcohol gradient. Later, the shoot sections were embedded in liquid paraffin wax (temperature $56-58^{\circ} \mathrm{C}$ ). The shoot sections $(15 \mu \mathrm{m})$ for optical microscopy observations were sliced by a microtome. Each time point was represent by at least five images per sample.

The activities of beta-glucosidase (beta-GC), trehalose6-phosphate synthase (TPS), and beta-amylase (BAM) and the contents of malondialdehyde (MDA), hydrogen peroxide $\left(\mathrm{H}_{2} \mathrm{O}_{2}\right)$, soluble sugars, maltose, and trehalose were measured using corresponding assay kits according to the manufacturer's instructions (Suzhou Comin Bioengineering Co., Ltd., Suzhou, China). The experiments involved three biological replicates.

\section{RNA extraction and RNA-seq}

The samples were subjected to RNA extraction using an RNA Isolation Kit (Waryoung, China). Both the quality and concentration of the RNA samples were subsequently measured using an Agilent 2100 Bioanalyzer (Agilent Technologies, USA), a Qubit 3.0 Fluorometer (Invitrogen, USA), and a NanoDrop 2000 spectrophotometer (NanoDrop Products, USA). For Illumina libraries, the total RNA was subjected to mRNA purification using magnetic beads linked with oligo (dT) beads. The mRNA molecules were fragmented into short fragments, the short fragments were used as templates to synthesize cDNA, and the library was constructed. The library was subjected to $150 \mathrm{bp}$ paired-end sequencing using the NGS tool of the Illumina HiSeq 2500 platform (Illumina Inc., USA). For PacBio libraries, cDNA was prepared using a SMRTer PCR cDNA Synthesis Kit (Clontech, USA), and PacBio libraries were constructed according to the PacBio protocol (http://www.pacb.com/wpcontent).

\section{Data processing}

The short-read data were obtained via NGS, and raw reads were filtered by removing adapter reads, poly- $\mathrm{N}$ reads and low-quality reads to obtain clean reads [63]. The PacBio sequencing results were analyzed using the SMRT Pipe analysis workflow of the PacBio SMRT Analysis software suite (http://www.pacb.com/products-andservices/analytical-software/smrt-analysis). In brief, to obtain high-quality reads, the SMRT sequencing results were filtered by removing polymerase reads with a length of $<50 \mathrm{bp}$ and a quality of $<0.75$. The highquality reads were subsequently chosen to obtain errorcorrected circular consensus sequences (CCSs) for which the full passes were $\geq 0$ and the quality was $>0.75$. The CCSs were regarded as full-length (FL) transcripts when the $5^{\prime}$ primer sequence, 3 ' primer sequence, and a polyA tail were present. The artificial chimeric sequences were produced by direct linkages of two cDNA template strands due to the low concentrations of adapters or SMRTbell, whereas nonchimeric sequences in the FL sequence were considered to be FL-nonchimeric (FLNC) sequences. The FLNC transcripts were identified by searching for $5^{\prime}$ tail cDNA primers and poly-A tail signals in the CCSs. FLNC transcripts with a high-quality base were obtained for subsequent analysis.

\section{Functional annotations of transcripts and identification of DEGs}

Due to the absence of a reference genome for tetraploid kiwifruit, a reference transcriptome was assembled de novo from SMRT transcript data. Unigene sequences were annotated in various databases, and annotation information of the unigenes was obtained for those with an E-values of at least 1e-5. Five databases were used: the nonredundant protein sequence (NR) database, Gene Ontology (GO) database, Eukaryotic Ortholog Groups (KOG) database, Homologous Protein Family (Pfam) database, and Kyoto Encyclopedia of Genes and Genomes (KEGG) database. iTAK was used to predict plant transcription factors (TFs), and the TF family classification used by the database was adopted to identify TFs by hmmscan. The DESeq tool ( $\mathrm{R}$ package version 1.10.1) was used to identify the DEGs (differentially expressed genes) between different comparison groups, and filtered standards were adjusted according to $P$-value $<0.05$ and $\mid \log 2$ (fold-change) $\mid \geq 1$. Via KEGG and GO databases, the enriched DEGs were used to identify significant metabolic pathways, and the filtered standard was an FDR-adjusted $P$-value of $<0.05$.

\section{Validation and expression analysis of DEGs by quantitative reverse transcription-PCR (qRT-PCR)}

First-strand cDNA was synthesized from a total of $2 \mu \mathrm{g}$ of template RNA using a ReverTra Ace qPCR RT Kit (Toyobo, Osaka, Japan) according to the manufacturer's instructions. qRT-PCR was subsequently performed on a LightCycler 480 real-time PCR system (Roche, Basel, Switzerland) using 2x SYBR Green I Master Mix (Asbio Technology, Inc.) and the first-strand cDNA template. The qRT-PCR protocol was as follows: $95^{\circ} \mathrm{C}$ for $5 \mathrm{~min}$, followed by 45 cycles of denaturation at $95^{\circ} \mathrm{C}$ for $10 \mathrm{~s}$, annealing at $60^{\circ} \mathrm{C}$ for $20 \mathrm{~s}$, and extension at $72^{\circ} \mathrm{C}$ for 20 $\mathrm{s}$. The relative expression levels of each sample were calculated in relation to the expression of the reference gene $\beta$-actin using the $2^{-\Delta \Delta C T}$ method. Three independent biological replications were included. All the primers used in this study are shown in Table S5.

\section{Gene coexpression network analysis and network visualization}

Weighted gene coexpression network analysis (WGCN A) was performed using the WGCNA package ( $R$ 
package version 1.69) with the default parameters. Using the FPKM values of the DEGs, an adjacency matrix was constructed. The resulting adjacency matrix was then converted into a topological overlap matrix (TOM). A dynamic hybrid tree-cut algorithm (via the $\mathrm{R}$ package dynamicTreeCut, v.1.63-1) was used to detect modules, with the default settings. The phenotypic data were used to identify the related modules, and a correlation matrix between phenotypes and gene modules was constructed using the WGCNA package. The genes from the related module were visualized using Cytoscape (v.3.8.2).

\section{Statistical analyses}

Two-way analysis of variance (ANOVA) accompanying Tukey's test was performed for all data using SPSS 22 (IBM, Inc., Armonk, NY, USA) and Prism 7 (GraphPad Software, Inc., La Jolla, CA, USA). Each experiment was repeated at least three times independently, and Pearson's one-tailed test was conducted at the $P<0.05$ significance level.

\section{Supplementary Information}

The online version contains supplementary material available at https://doi. org/10.1186/s12870-021-03152-w.

\section{Additional file 1 Supplementary Fig. 1. GO functional classifications}

of all the unigenes.

Additional file 2 Supplementary Fig. 2. KEGG functional

classifications of all the unigenes.

Additional file $\mathbf{3}$ Supplementary Fig. 3. Phylogenetic tree of candidate genes and homologous genes. (a) Beta-GC, (b) TPS5, (c) BAM3.1, (d) CBF3, (e) MYC2, and (f) MYB44.

Additional file 4 Table S1. DEGs related to Starch and sucrose metabolism, MAPK signaling pathway, Phosphatidylinositol signaling system, Inositol phosphate metabolism, Arginine and proline metabolism.

Additional file 5 Table S2. DEGs related to hormone and calcium signal.

Additional file 6 Table S3. DEGs related to TFs.

Additional file $\mathbf{7}$ Table S4. Top 100 hub genes in the blue module. Additional file $\mathbf{8}$ Table S5. Primers used for quantitative real-time RTPCR analysis.

\section{Acknowledgements}

Not applicable.

\section{Authors' contributions}

SS, ML, XQ JC, HG, YZ, LS, AM and DB conducted the experiments; JF organized and supervised the overall project; SS and ML performed the data analysis and wrote the manuscript writing; and $\mathrm{CH}$ edited the manuscript. All authors have read and approved the manuscript.

\section{Funding}

This study was funded by the National Key Research and Development Project of China (Grant No. 2019YFD1000200); the National Science Foundation of China (Grant No. 31801820); the Special Engineering Science and Technology Innovation, Chinese Academy of Agricultural Sciences (Grant No. CAAS-ASTIP-2015-ZFRI); and the Modern Agricultural Industrial Technology System of Henan Province (Grant No. S2014-11). The funders had no role in study design, collection, analysis, and interpretation of data and in writing the manuscript, but just provide the financial support.

\section{Availability of data and materials}

The raw data generated in this study have been deposited in the NCBI Short Read Archive (SRA) under the accession number PRJNA681641. All other relevant data contained within the paper are available in the paper and Supplementary Files.

\section{Declarations}

Ethics approval and consent to participate

Not applicable.

Consent for publication

Not applicable.

\section{Competing interests}

The authors declare that they have no conflicts of interest concerning this work.

Received: 1 April 2021 Accepted: 2 August 2021

Published online: 11 August 2021

\section{References}

1. Sun XM, Zhang LL, Wong DCJ, Wang Y, Zhu ZF, Xu GZ, et al. The ethylene response factor VaERF092 from Amur grape regulates the transcription factor VaWRKY33, improving cold tolerance. Plant J. 2019;99(5):988-1002. https://doi.org/10.1111/tpj.14378.

2. Zhang LL, Zhao TT, Sun XM, Wang Y, Du C, Zhu ZF, et al. Overexpression of VaWRKY12, a transcription factor from Vitis amurensis with increased nuclear localization under low temperature, enhances cold tolerance of plants. Plant Mol Biol. 2019;100(1-2):95-110. https://doi.org/10.1007/s11103019-00846-6.

3. Raju SKK, Barnes AC, Schnable JC, Roston RL. Low-temperature tolerance in land plants: are transcript and membrane responses conserved? Plant Sci. 2018;276:73-86. https://doi.org/10.1016/j.plantsci.2018.08.002.

4. Wisniewski M, Nassuth A, Arora R. Cold hardiness in trees: a Mini-review. Front Plant Sci. 2018;9:1394. https://doi.org/10.3389/fpls.2018.01394.

5. Shen JZ, Zhang DY, Zhou L, Zhang XZ, Liao JR, Duan Y, et al. Transcriptomic and metabolomic profiling of Camellia sinensis L. cv. 'Suchazao' exposed to temperature stresses reveals modification in protein synthesis and photosynthetic and anthocyanin biosynthetic pathways. Tree Physiol. 2019; 39(9):1583-99. https://doi.org/10.1093/treephys/tpz059.

6. Singh AK, Dhanapal S, Yadav BS. The dynamic responses of plant physiology and metabolism during environmental stress progression. Mol Biol Rep. 2020:47(2):1459-70. https://doi.org/10.1007/s11033-019-05198-4.

7. Yue C, Cao HL, Wang L, Zhou YH, Huang YT, Hao XY, et al. Effects of cold acclimation on sugar metabolism and sugar-related gene expression in tea plant during the winter season. Plant Mol Biol. 2015;88(6):591-608. https:// doi.org/10.1007/s11103-015-0345-7.

8. He Z, Zhao T, Yin Z, Liu J, Cheng Y, Xu J. The phytochrome-interacting transcription factor CSPIF8 contributes to cold tolerance in citrus by regulating superoxide dismutase expression. Plant Sci. 2020;298:110584. https://doi.org/10.1016/j.plantsci.2020.110584.

9. Ming $R$, Zhang $Y$, Wang $Y$, Khan M, Dahro B, Liu J-H. The JA-responsive MYC2-BADH-like transcriptional regulatory module in Poncirus trifoliata contributes to cold tolerance by modulation of glycine betaine biosynthesis. New Phytol. 2021;229(5):2730-50.

10. Wang $Y$, Xin H, Fan P, Zhang J, Liu Y, Dong Y, et al. The genome of Shanputao (Vitis amurensis) provides a new insight into cold tolerance of grapevine. Plant J. 2021;105(6):1495-506.

11. Huang SX, Ding J, Deng DJ, Tang W, Sun HH, Liu DY, et al. Draft genome of the kiwifruit Actinidia chinensis. Nat Commun. 2013;4(1):2640. https://doi. org/10.1038/ncomms3640.

12. Pilkington SM, Crowhurst R, Hilario E, Nardozza S, Fraser L, Peng YY, et al. A manually annotated Actinidia chinensis var. chinensis (kiwifruit) genome highlights the challenges associated with draft genomes and gene prediction in plants. BMC Genomics. 2018;19:19.

13. Tang W, Sun XP, Yue JY, Tang XF, Jiao C, Yang Y, et al. Chromosome-scale genome assembly of kiwifruit Actinidia eriantha with single-molecule sequencing and chromatin interaction mapping. GigaScience. 2019:8(4):10. 
14. Wu H, Ma T, Kang M, Ai F, Zhang J, Dong G, et al. A high-quality Actinidia chinensis (kiwifruit) genome. Horticulture Res. 2019;6(1):117. https://doi. org/10.1038/s41438-019-0202-y.

15. Wu QY, Wang JW, Mao SX, Xu HR, Wu Q, Liang MT, et al. Comparative transcriptome analyses of genes involved in sulforaphane metabolism at different treatment in Chinese kale using full-length transcriptome sequencing. BMC Genomics. 2019;20:377.

16. Lou HQ, Ding MZ, Wu JS, Zhang FC, Chen WC, Yang Y, et al. Full-length transcriptome analysis of the genes involved in tocopherol biosynthesis in Torreya grandis. J Agric Food Chem. 2019;67(7):1877-88. https://doi.org/10.1 021/acs.jafc.8b06138.

17. Xu ZC, Peters RJ, Weirather J, Luo HM, Liao BS, Zhang X, et al. Full-length transcriptome sequences and splice variants obtained by a combination of sequencing platforms applied to different root tissues of Salvia miltiorrhiza and tanshinone biosynthesis. Plant J. 2015;82(6):951-61. https://doi.org/1 $0.1111 /$ tpj.12865.

18. Li YP, Dai C, Hu CG, Liu ZC, Kang CY. Global identification of alternative splicing via comparative analysis of SMRT- and Illumina-based RNA-seq in strawberry. Plant J. 2017;90(1):164-76. https://doi.org/10.1111/tpj.13462.

19. He L, Fu SH, Xu ZC, Yan J, Xu J, Zhou H, et al. Hybrid sequencing of fulllength CDNA transcripts of stems and leaves in Dendrobium officinale. Genes. 2017;8(10):257. https://doi.org/10.3390/genes8100257.

20. Wang XM, Chen SY, Shi X, Liu DN, Zhao P, Lu YZ, et al. Hybrid sequencing reveals insight into heat sensing and signaling of bread wheat. Plant $J$. 2019;98(6):1015-32. https://doi.org/10.1111/tpj.14299.

21. Minio A, Massonnet M, Figueroa-Balderas R, Vondras AM, Blanco-Ulate B, Cantu D. Iso-Seq Allows Genome-Independent Transcriptome Profiling of Grape Berry Development. G3. 2019;9(3):755-67.

22. Dong LL, Liu HF, Zhang JC, Yang SJ, Kong GY, Chu JSC, et al. Singlemolecule real-time transcript sequencing facilitates common wheat genome annotation and grain transcriptome research. BMC Genomics. 2015;16(1):1039. https://doi.org/10.1186/s12864-015-2257-y.

23. Ma XS, Xia H, Liu YH, Wei HB, Zheng XG, Song CZ, et al. Transcriptomic and Metabolomic studies disclose key metabolism pathways contributing to well-maintained photosynthesis under the drought and the consequent drought-tolerance in Rice. Front Plant Sci. 2016;7:1886.

24. Luo HT, Zhang JY, Wang G, Jia ZH, Huang SN, Wang T, et al. Functional characterization of waterlogging and heat stresses tolerance gene pyruvate decarboxylase 2 from Actinidia deliciosa. Int J Mol Sci. 2017;18(11):2377. https://doi.org/10.3390/ijms18112377.

25. Ho WWH, Hill CB, Doblin MS, Shelden MC, van de Meene A, Rupasinghe T, et al. Integrative multi-omics analyses of barley Rootzones under salinity stress reveal two distinctive salt tolerance mechanisms. Plant Commun. 2020;1(3):100031. https://doi.org/10.1016/j.xplc.2020.100031.

26. Solanke AU. Signal transduction during cold stress in plants. Physiol Mol Biol Plants. 2008;14(1/2):69-79. https://doi.org/10.1007/s12298-008-0006-2.

27. Mizoi J, Shinozaki K, Yamaguchi-Shinozaki K. AP2/ERF family transcription factors in plant abiotic stress responses. BBA Gene Regul Mech. 2012; 1819(2):86-96.

28. Xie YP, Chen PX, Yan Y, Bao CN, Li XW, Wang LP, et al. An atypical R2R3 MYB transcription factor increases cold hardiness by CBF-dependent and CBF-independent pathways in apple. New Phytol. 2018;218(1):201-18. https://doi.org/10.1111/nph.14952.

29. Li H, Ding YL, Shi YT, Zhang XY, Zhang SQ, Gong ZZ, et al. MPK3-and MPK6mediated ICE1 phosphorylation negatively regulates ICE1 stability and freezing tolerance in Arabidopsis. Dev Cell. 2017;43(5):630-42. https://doi. org/10.1016/j.devcel.2017.09.025.

30. Rubio S, Noriega X, Perez FJ. Abscisic acid (ABA) and low temperatures synergistically increase the expression of CBF/DREB1 transcription factors and cold-hardiness in grapevine dormant buds. Ann Bot. 2019;123(4):681-9. https://doi.org/10.1093/aob/mcy201.

31. Tang K, Zhao L, Ren YY, Yang SH, Zhu JK, Zhao CZ. The transcription factor ICE1 functions in cold stress response by binding to the promoters of CBF and COR genes. J Integr Plant Biol. 2020;62(3):258-63. https://doi.org/1 $0.1111 / j i p b .12918$.

32. Kou S, Chen L, Tu W, Scossa F, Wang YM, Liu J, et al. The arginine decarboxylase gene ADC1, associated to the putrescine pathway, plays an important role in potato cold-acclimated freezing tolerance as revealed by transcriptome and metabolome analyses. Plant J. 2018;96(6):1283-98. https://doi.org/10.1111/tpj.14126.
33. Cheong BE, Ho WWH, Biddulph B, Wallace X, Rathjen T, Rupasinghe TWT, et al. Phenotyping reproductive stage chilling and frost tolerance in wheat using targeted metabolome and lipidome profiling. Metabolomics. 2019; 15(11):144. https://doi.org/10.1007/s11306-019-1606-2.

34. Yang M, Yang J, Su L, Sun K, Li DX, Liu YZ, et al. Metabolic profile analysis and identification of key metabolites during rice seed germination under low-temperature stress. Plant Sci. 2019;289:110282. https://doi.org/10.1016/j. plantsci.2019.110282

35. Sun S, Qi X, Wang R, Lin M, Fang J. Evaluation of freezing tolerance inActinidiagermplasm based on relative electrolyte leakage. Hortic Environ Biotechnol. 2020;61(4):755-65. https://doi.org/10.1007/s13580-020-00272-4

36. Thalmann $M$, Santelia D. Starch as a determinant of plant fitness under abiotic stress. New Phytol. 2017;214(3):943-51. https://doi.org/10.1111/ nph.14491.

37. Ouyang L, Leus L, De Keyser E, Van Labeke M-C. Seasonal changes in cold hardiness and carbohydrate metabolism in four garden rose cultivars. J Plant Physiol. 2019;232:188-99. https://doi.org/10.1016/j.jplph.2018.12.001.

38. $\mathrm{Xu} Z \mathrm{Z}$, Lee $\mathrm{KH}$, Dong T, Jeong JC, Jin JB, Kanno Y, et al. A vacuolar $\beta$ glucosidase homolog that possesses glucose-conjugated abscisic acid hydrolyzing activity plays an important role in osmotic stress responses in Arabidopsis. Plant Cell. 2012;24(5):2184-99. https://doi.org/10.1105/tpc.112. 095935

39. Akiyama T, Opassiri R, Cairns JK. A rice homolog of Arabidopsis sfr2 gene encoding beta-glucosidase is responsive to cold stress. Plant Cell Physiol. 2007;48:S237.

40. Thorlby G. The SENSITIVE TO FREEZING2 gene, required for FREEZING tolerance in Arabidopsis thaliana, encodes a beta-glucosidase. Plant Cell. 2004;16(8):2192-203. https://doi.org/10.1105/tpc.104.024018.

41. Opassiri R, Pomthong B, Akiyama T, Nakphaichit M, Onkoksoong T, Cairns MK, et al. A stress-induced rice (Oryza sativa L.) beta-glucosidase represents a new subfamily of glycosyl hydrolase family 5 containing a fascin-like domain. Biochem J. 2007:408(2):241-9. https://doi.org/10.1042/BJ20070734.

42. Khazaei M, Maali-Amiri R, Talei AR, Ramezanpour S. Differential transcript accumulation of Dhydrin and Beta-glucosidase genes to cold-induced oxidative stress in chickpea. J Agric Sci Technol. 2015;17(3):725-34.

43. Wang PT, Liu H, Hua HJ, Wang L, Song CP. A vacuole localized betaglucosidase contributes to drought tolerance in Arabidopsis. Chin Sci Bull. 2011;56(33):3538-46. https://doi.org/10.1007/s11434-011-4802-7.

44. Benaroudj N, Lee DH, Goldberg AL. Trehalose accumulation during cellular stress protects cells and cellular proteins from damage by oxygen radicals. J Biol Chem. 2001;276(26):24261-7. https://doi.org/10.1074/jbc.M101487200.

45. Fernandez O, Béthencourt L, Quero A, Sangwan RS, Clément C. Trehalose and plant stress responses: friend or foe? Trends Plant Sci. 2010;15(7):40917. https://doi.org/10.1016/j.tplants.2010.04.004.

46. Mostofa MG, Hossain MA, Fujita M, Tran LSP. Physiological and biochemical mechanisms associated with trehalose-induced copper-stress tolerance in rice. Sci Rep. 2015;5(1):11433. https://doi.org/10.1038/srep11433.

47. Li HW, Zang BS, Deng XW, Wang XP. Overexpression of the trehalose-6phosphate synthase gene OSTPS1 enhances abiotic stress tolerance in rice. Planta. 2011;234(5):1007-18. https://doi.org/10.1007/s00425-011-1458-0.

48. Ge LF, Chao DY, Shi M, Zhu MZ, Gao JP, Lin HX. Overexpression of the trehalose-6-phosphate phosphatase gene OsTPP1 confers stress tolerance in rice and results in the activation of stress responsive genes. Planta. 2008; 228(1):191-201. https://doi.org/10.1007/s00425-008-0729-x.

49. $X L A, L F A, P Q A, Y S A, J L B, X W$ A. Overexpression of the wheat trehalose 6 - phosphate synthase 11 gene enhances cold tolerance in Arabidopsis thaliana. Gene. 2019;710:210-7. https://doi.org/10.1016/j.gene.2019.06.006.

50. Bae H, Herman E, Bailey B, Ho B, Sicher R. Exogenous trehalose alters Arabidopsis transcripts involved in cell wall modification, abiotic stress, nitrogen metabolism, and plant defense. Physiol Plant. 2010;125(1):114-26.

51. Ding YL, Shi YT, Yang SH. Advances and challenges in uncovering cold tolerance regulatory mechanisms in plants. New Phytol. 2019;222(4):1690704. https://doi.org/10.1111/nph.15696.

52. Valerio C, Costa A, Marri L, Issakidis-Bourquet E, Pupillo P, Trost $P$, et al. Thioredoxin-regulated -amylase (BAM1) triggers diurnal starch degradation in guard cells, and in mesophyll cells under osmotic stress. J Exp Bot. 2011; 62(2):545-55. https://doi.org/10.1093/jxb/erq288.

53. Monroe JD, Storm AR. Review: the Arabidopsis $\beta$-amylase (BAM) gene family: diversity of form and function. Plant Sci. 2018;276:163-70. https://doi. org/10.1016/j.plantsci.2018.08.016. 
54. Sun S, Fang J, Lin M, Qi X, Chen J, Wang R, et al. Freezing tolerance and expression of beta-amylase gene in two Actinidia arguta cultivars with seasonal changes. Plants. 2020;9(4):1-12.

55. Zhao LY, Yang TY, Xing CH, Dong HZ, Qi KJ, Gao JZ, et al. The beta-amylase PbrBAM3 from pear (Pyrus betulaefolia) regulates soluble sugar accumulation and ROS homeostasis in response to cold stress. Plant Sci. 2019;287:110184. https://doi.org/10.1016/j.plantsci.2019.110184.

56. Peng T, Zhu XF, Duan N, Liu JH. PtrBAM1, a beta-amylase-coding gene of Poncirus trifoliata, is a CBF regulon member with function in cold tolerance by modulating soluble sugar levels. Plant Cell Environ. 2014;37(12):2754-67. https://doi.org/10.1111/pce.12384.

57. Zakharian E, Cao C, Rohacs T. Gating of transient receptor potential Melastatin 8 (TRPM8) channels activated by cold and chemical agonists in planar lipid bilayers. J Neurosci. 2010;30(37):12526-34. https://doi.org/10.1 523/JNEUROSCI.3189-10.2010.

58. Liu YN, Lu XX, Ren A, Shi L, Zhu J, Jiang AL, et al. Conversion of phosphatidylinositol (PI) to PI4-phosphate (PI4P) and then to PI $(4,5) \mathrm{P}$-2 is essential for the cytosolic Ca2+ concentration under heat stress in Ganoderma lucidum. Environ Microbiol. 2018;20(7):2456-68. https://doi. org/10.1111/1462-2920.14254.

59. Wimalasekera P, Holk M, Scherer GFE. Plant Phosphatidylcholine-Hydrolyzing Phospholipases C NPC3 and NPC4 with Roles in Root Development and Brassinolide Signaling in Arabidopsis thaliana RID A-7853-2010. Mol Plant. 2010;3(3):610-25.

60. Kravets VS, Kolesnikov YS, Kretynin SV, Kabachevskaya EM, Kukhar VP. Molecular and genetics approaches for investigation of phospholipase D role in plant cells. Biopolym Cell. 2010;26(3):175-86. https://doi.org/10.7124/ bc.000154.

61. Smart CC, Fleming AJ. A plant gene with homology to D-myo-inositol-3phosphate synthase is rapidly and spatially up-regulated during an abscisicacid-induced morphogenic response in Spirodela polyrrhiza. Plant J. 2010; 4(2):279-93.

62. Smart CC, Flores S. Overexpression of D-myo-inositol-3-phosphate synthase leads to elevated levels of inositol in Arabidopsis. Plant Mol Biol. 1997;33(5): 811-20. https://doi.org/10.1023/A:1005754425440.

63. Peng T, You XS, Guo L, Zhong BL, Mi LF, Chen JM, et al. Transcriptome analysis of Chongyi wild mandarin, a wild species more cold-tolerant than Poncirus trifoliata, reveals key pathways in response to cold. Environ Exp Bot. 2021;184:104371. https://doi.org/10.1016/j.envexpbot.2020.104371.

64. Zhang CQ, Wang J, Gao X. Computational identification of transcriptional regulatory elements in Arabidopsis TCH4 promoter. Hereditas. 2008;30(5): 620-6. https://doi.org/10.3724/sp.j.1005.2008.00620.

65. Wang J, Ren Y, Liu X, Luo S, Zhang X, Liu X, et al. Transcriptional activation and phosphorylation of OsCNGC9 confer enhanced chilling tolerance in rice. Mol Plant. 2021;14(2):315-29.

66. Zhao CZ, Wang PC, Si T, Hsu CC, Wang L, Zayed O, et al. MAP kinase cascades regulate the cold response by modulating ICE1 protein stability. Dev Cell. 2017;43(5):618-29. https://doi.org/10.1016/j.devcel.2017.09.024.

67. Tang W, Tang AY. Overexpression of Arabidopsis thaliana malonyl-CoA synthetase gene enhances cold stress tolerance by activating mitogenactivated protein kinases in plant cells. J Forestry Res. 2021;32(2):741-53.

68. Meng S, Dane F, Si Y, Ebel R, Zhang C. Gene expression analysis of cold treated versus cold acclimated Poncirus trifoliata. Euphytica. 2008;164(1): 209-19. https://doi.org/10.1007/s10681-008-9708-3.

69. Shi YT, Ding YL, Yang SH. Molecular Reculation of CBF Sicnalinc in Colc acclimation. Trends Plant Sci. 2018;23(7):623-37. https://doi.org/10.1016/j. tplants.2018.04.002.

70. Wang YJ, Zhang ZG, He XJ, Zhou HL, Wen YX, Dai JX, et al. A rice transcription factor OsbHLH1 is involved in cold stress response. Theor Appl Genet. 2003;107(8):1402-9. https://doi.org/10.1007/s00122-003-1378-X.

71. Feng XM, Zhao Q, Zhao LL, Qiao Y, Xie XB, Li HF, et al. The cold-induced basic helix-loop-helix transcription factor gene MdClbHLH1 encodes an ICElike protein in apple. BMC Plant Biol. 2012;12(1):22. https://doi.org/10.11 86/1471-2229-12-22

72. Wang $X$, Ding Y, Li Z, Shi Y, Wang J, Hua J, et al. PUB25 and PUB26 promote plant freezing tolerance by degrading the cold signaling negative regulator MYB15. Dev Cell. 2019;51(2):222-35. https://doi.org/10.1016/j.devcel.2019.08. 008.

73. Sun MY, Li JY, Li D, Huang FJ, Wang D, Li H, et al. Full-length transcriptome sequencing and modular organization analysis of the Naringin/
Neoeriocitrin-related gene expression pattern in Drynaria roosii. Plant Cell Physiol. 2018;59(7):1398-414. https://doi.org/10.1093/pcp/pcy072.

74. Umer MJ, Bin Safdar L, Gebremeskel H, Zhao S, Yuan P, Zhu H, et al. Identification of key gene networks controlling organic acid and sugar metabolism during watermelon fruit development by integrating metabolic phenotypes and gene expression profiles. Hortic Res. 2020;7(1):193. https:// doi.org/10.1038/s41438-020-00416-8.

75. Nakata M, Sugiyama N, Pankasemsuk T. Morphological study of the structure and development of longan inflorescence. J Am Soc Hortic Sci. 2005;130(6):793-8. https://doi.org/10.21273/JASHS.130.6.793.

\section{Publisher's Note}

Springer Nature remains neutral with regard to jurisdictional claims in published maps and institutional affiliations.
Ready to submit your research? Choose BMC and benefit from:

- fast, convenient online submission

- thorough peer review by experienced researchers in your field

- rapid publication on acceptance

- support for research data, including large and complex data types

- gold Open Access which fosters wider collaboration and increased citations

- maximum visibility for your research: over $100 \mathrm{M}$ website views per year

At BMC, research is always in progress.

Learn more biomedcentral.com/submissions 